\title{
1 Evolution of mouse circadian enhancers from transposable elements
}

2

3 Julius Judd ${ }^{1}$, Hayley Sanderson ${ }^{2}$, and Cédric Feschotte ${ }^{1 *}$

4

$5 \quad{ }^{1}$ Department of Molecular Biology and Genetics, Cornell University, Ithaca, New York 14853, 6 USA

$7 \quad{ }^{2}$ Department of Human Genetics, University of Utah School of Medicine, Salt Lake City, Utah, 8 84112, USA

$9 \quad{ }^{*}$ Correspondence: cf458@cornell.edu

10

11

ORCID: 0000-0002-4602-0205 (JJ); 0000-0002-8772-6976 (CF) 


\section{Abstract}

\section{Background}

15 Transposable elements are increasingly recognized as a source of cis-regulatory variation.

16 Previous studies have revealed that transposons are often bound by transcription factors and

17 some have been co-opted into functional enhancers regulating host gene expression. However,

18 the process by which transposons mature into complex regulatory elements, like enhancers,

19 remains poorly understood. To investigate this process, we examined the contribution of

20 transposons to the cis-regulatory network controlling circadian gene expression in the mouse

21 liver, a well-characterized network serving an important physiological function.

\section{Results}

23 ChIP-seq analyses revealed that transposons and other repeats contribute $\sim 14 \%$ of the binding

24 sites for core circadian regulators (CRs) including BMAL1, CLOCK, PER1/2, and CRY1/2, in

25 the mouse liver. RSINE1, an abundant murine-specific SINE, was the only transposon family

26 enriched for CR binding sites across all datasets. Sequence analyses and reporter assays revealed

27 that the circadian regulatory activity of RSINE1 stems from the presence of imperfect CR

28 binding motifs in the ancestral RSINE1 sequence. These motifs matured into canonical motifs

29 through point mutations after transposition. Furthermore, maturation occurred preferentially

30 within elements inserted in proximity of ancestral CR binding sites. RSINE1 also acquired

31 motifs that recruit nuclear receptors known to cooperate with CR to regulate circadian gene

32 expression specifically in the liver.

\section{Conclusions}

34 Our results suggest that the birth of enhancers from transposons is predicated both by the sequence 35 of the transposon and by the cis-regulatory landscape surrounding their genomic integration site. 
bioRxiv preprint doi: https://doi.org/10.1101/2020.11.09.375469; this version posted November 10,2020 . The copyright holder for this preprint (which was not certified by peer review) is the author/funder, who has granted bioRxiv a license to display the preprint in perpetuity. It is made available under aCC-BY 4.0 International license.

36 This model illuminates how transposition fuels the emergence and turnover of enhancers during

37 mammalian evolution.

38

39 Keywords: Transposable elements, enhancers, transcription, gene regulation, regulatory

40 evolution, circadian rhythms

41 


\section{Background}

Change in gene regulation is an important mechanism underlying the emergence of new

44 biological traits [1-5]. There is a substantial body of empirical studies illustrating how the

45 addition, modification, or disappearance of cis-regulatory elements, such as enhancers, has

46 driven the emergence of profound phenotypic changes throughout evolution [6-8]. Thus, there

47 has been an intensifying effort over the past decade to better understand mechanisms underlying

48 the evolution of enhancers and other cis-regulatory elements [4,9-12].

In the broadest definition, enhancers are short (100 bp-1 kb) DNA sequences that

51 modulate transcription of target genes regardless of genomic orientation or distance, and are

52 often bound by transcription factors (TFs) [13,14]. Recent advances in functional genomics

53 enabled nearly unbiased mapping of enhancers and their associated TF binding sites (TFBSs) on

54 a genome-wide scale and facilitated systematic studies of enhancer evolution across and within

55 species [11,15-17]. Seminal comparative studies in mammals revealed a low level of

56 conservation in the genomic location of enhancers relative to genes and their promoters [18-27].

57 For instance, Villar and colleagues found that nearly half of 20,000-25,000 active liver

58 enhancers mapped in each of 20 mammalian species are lineage- or even species-specific, while

59 almost all promoters active in the liver are conserved across most or all the species examined

60 [28]. However, recent analyses demonstrated that deeply conserved enhancers often coordinate

61 robust and essential gene expression programs while less conserved enhancers contribute

62 plasticity and redundancy to gene regulatory networks [29-31]. While these studies point to the

63 rapid turnover of enhancers during mammalian evolution, the mechanisms underlying the birth

64 and death of enhancers are only beginning to be understood [10,11,26,32-35]. 
67 variation in virtually all metazoans [36]. For example, between one and two thirds of all

68 mammalian genomes thus far examined are recognizable as being derived from TE sequences

69 [36-39]. These elements inserted at various times during mammalian evolution, ranging from

70 highly decayed copies integrated $>100$ million years ago to recently integrated copies that may

71 be species-specific or still polymorphic in the population [37-42]. Several studies have

72 systematically examined the contribution of TEs to TF binding and the birth of cis-regulatory

73 elements, and some general principles have emerged [43-47]. First, TEs contribute a substantial

74 but widely variable fraction $(\sim 2-40 \%)$ of the TFBSs mapped for a given TF throughout the

75 genome [48-52]. Second, TFBSs and cis-regulatory elements derived from TEs tend to be

76 evolutionarily recent, and are restricted to specific species or lineages $[34,50,53]$. For example,

$77 \sim 20 \%$ of OCT4 and NANOG binding sites were derived from lineage-specific TEs in humans

78 and mice [20]. This may be explained by the fact that the majority of TEs in any mammalian

79 genome are themselves lineage-specific: for example, $85 \%$ of mouse TEs are not shared with the

80 human [40] and 35\% are not even shared with the rat [54]. Third, not all TEs contribute equally:

81 for any given TF there is generally one or a few TE families that account for a disproportionate

82 fraction of binding sites relative to their frequency in the genome [20,44,46,48,50,51].

Multiple studies have now confirmed that different TE classes and families contribute

85 TFBSs for different TFs in different mammalian species, and that these TE-derived TFBSs

86 occasionally undergo exaptation to give rise to new host regulatory elements (reviewed in

$87[44,47])$. However, the mechanisms by which complex enhancers emerge from TEs remain 
88 poorly understood. For instance, it is unclear why specific TE families or copies are bound by a

89 particular TF while closely related elements in the same genome are not $[45,51]$. The path by

90 which individual TE copies are co-opted for regulatory purposes has been scarcely characterized

91 [55], and the relative contributions of combinatorial sequence motifs pre-existing within TE or in

92 the vicinity of their insertion site, have not been examined in detail. To address these and other

93 poorly understood aspects of TE co-option in regulatory evolution, we chose to examine their

94 contribution to the cis-regulatory network underlying circadian gene expression. The machinery

95 responsible for the transcriptional control of circadian gene expression is deeply conserved and

96 has been extensively characterized in the mouse liver, which provides a solid experimental

97 framework against which the impact of TEs can be queried. The circadian clock also presents the

98 relatively unique advantage of providing a particularly robust system to examine the binding of

99 TEs by regulatory proteins, as circadian rhythms are maintained by a series of interconnecting

100 feedback loops of paralogous TFs [56-58].

101

The primary feedback loop consists of six circadian regulators (CRs), of which two are

103 transcriptional activators (BMAL1 and CLOCK) and four are transcriptional repressors (PER1,

104 PER2, CRY1 and CRY2). During the day, BMAL1 and CLOCK form a heterodimer, which

105 binds to a tandem pair of E-box motifs in distal and promoter regions of clock-controlled genes

106 [59]. Among the direct targets of the BMAL1:CLOCK complex are the repressors PER1/2 and

107 CRY1/2. Following translation, PER and CRY enter the nucleus and inhibit BMAL1:CLOCK

108 mediated transcription, thereby decreasing their own transcription and generating a feedback

109 loop essential to the maintenance of the clock period $[60,61]$. This model has recently been

110 revised to reflect reports that BMAL1 acts as a pioneer factor and promotes rhythmic 
111 nucleosome removal, and that transcription promoted by CLOCK:BMAL1 is not homogeneously

112 oscillatory [62]. It is proposed that CLOCK:BMAL1 binding rhythmically maintains a chromatin

113 landscape which facilitates binding and transcriptional activation by other ubiquitous or tissue-

114 specific transcription factors, including members of the nuclear receptor (NR) family [63].

115 Interactions between CRs and liver-specific NRs are thought to underlie liver-specific circadian

116 regulation of metabolic processes such as glucose, cholesterol, and lipid metabolism [64-66].

117 The vast amount of data and knowledge available for circadian regulation in the mouse liver

118 provides a solid paradigm to dissect the mechanisms underlying the contribution of particular

119 TEs to this cis-regulatory network. 


\section{Results}

122 Repetitive elements contribute CR TFBSs with weaker enhancer chromatin signatures

\section{3 than non-repetitive CR TFBSs}

To investigate the contribution of DNA repeats to cis-regulatory elements entwined in the

125 mammalian circadian gene regulatory network, we turned to a seminal collection of ChIP-seq

126 experiments that reported the genome-wide binding profiles of the six core CRs in the mouse

127 liver [67]. To utilize the most recent mouse genome annotation, we aligned raw sequencing reads

128 (Table 1) to the mm10 genome assembly and used uniquely mapped reads to call peaks. Of the

129 peaks identified with this approach, $\sim 60 \%-92 \%$ overlapped with peaks originally reported [67]

130 (Fig. S1A). Cognate sets of CLOCK and BMAL1 peaks overlapped by $\sim 85 \%$ (Fig. S1A). The

131 fraction of peaks that did not overlap between the two sets (based on their distance from each

132 other) were largely low confidence peaks (Fig. S1B). The fold change over background of the re-

133 called peaks correlated with the summit height of the original peaks (Fig. S1C). Overall, the total

134 number of peaks called for each CR was in good agreement with those reported in the published 135 analysis [67] (Fig. S1D). Together these findings validate this set of CR binding sites in the 136 mouse liver.

$\begin{array}{ccc}\text { Data } & \text { SRA } & \text { Citation } \\ \text { CLOCK, BMAL1, CRY1, CRY2, PER1, PER2 ChIP-seq } & \text { SRP014752 } & {[67]} \\ \text { DNase-seq, H3K27Ac ChIP-seq, Pol II ChIP-seq } & \text { SRP045509 } & {[68]} \\ \text { GRO-seq, ROR } \alpha \text { ChIP-seq } & \text { SRP044381 } & {[69]} \\ \text { RXR, LXR, PPAR } \alpha \text { ChIP-seq } & \text { SRP010657 } & {[70]}\end{array}$


REV-ERB $\alpha$, REV-ERB $\beta$ ChIP-seq

High confidence CLOCK:BMAL1 binding sites

Tissue Specific BMAL1 binding sites

Hepa 1-6 RNA-seq
SRP009472

N/A

SRP132869

SRP059935

Table 1: Sources of data used in this study.

141 the ChIP-seq peak coordinates showed positional overlap of $>50 \%$ with a repetitive element

142 annotated by RepeatMasker, which was accessed from the UCSC genome browser and filtered

143 for low complexity and simple repeats [74-76]. This analysis revealed that between $8.3 \%-14.3 \%$

144 of each CR peak set mapped within TEs or other repetitive elements (Fig. 1A). To corroborate

145 these results, we turned a published set of stringent CLOCK:BMAL1 binding sites that was

146 derived from the same ChIP-seq data but required occupancy by both CLOCK and BMAL1 and

147 was categorized as rhythmic, arrhythmic, or not expressed based on the circadian nascent

148 transcriptional output of the nearest gene [63]. Rhythmic peaks were further classified as

149 transcriptionally in or out of phase respective to CLOCK:BMAL1 binding. Interestingly, when

150 we intersected these peaks with the coordinates of repetitive elements as described above, we

151 observed slightly lower percentages $(6.3 \%-9.7 \%)$ of RABS (Fig. 1B), which led us to speculate

152 that TE-derived CR binding sites might be less likely to have biological relevance than non-

153 repeat-derived CR binding sites. 
156 binding profiles of each CR. As a control, we selected a random set of repeats matching the

157 familial composition of the RABS set. The binding pattern of CRs at RABS recapitulates that

158 observed at Non-RABS: CLOCK and BMAL1 binding increases from CT0-CT8 and then tapers

159 off, PER1/2 and CRY2 binding is initially low and increases from CT12-CT20, and CRY1

160 binding is maximally bound at CT0-CT4 but also increases at CT12 and CT20 (Fig.1C; Fig.

161 S2A). When we quantified ChIP-seq signal within peaks, the only statistically significant

162 difference consistent for all time points was that median CLOCK signal was increased in RABS

163 relative to Non-RABS. However, the magnitude of this increase is subtle and led us to conclude

164 that, overall, the oscillatory profile of CR binding at RABS and Non-RABS is essentially

165 indistinguishable. 
A

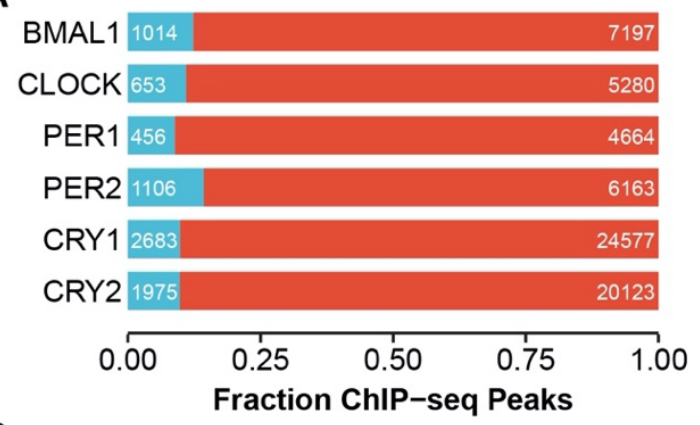

C

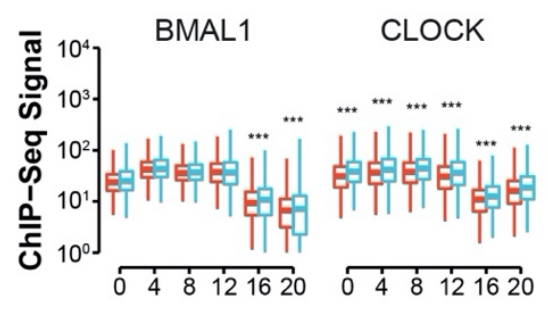

D
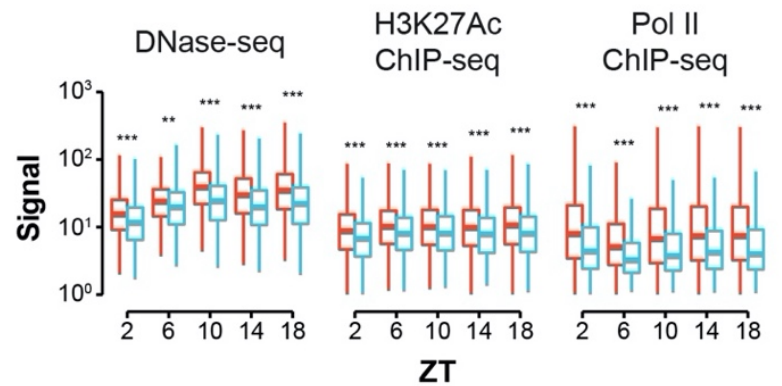

167

168

169

\section{B}

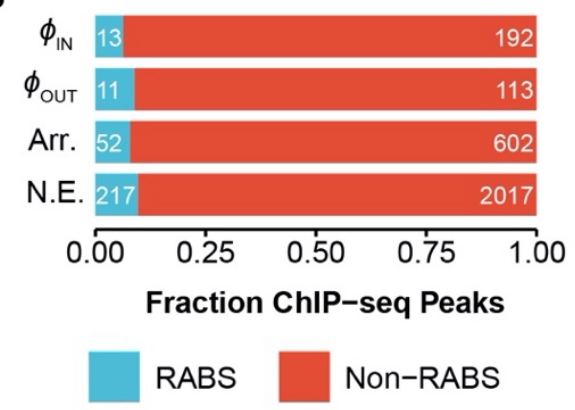

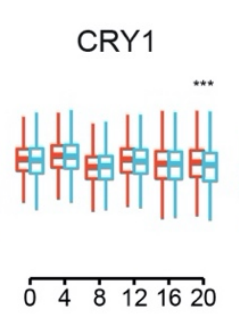
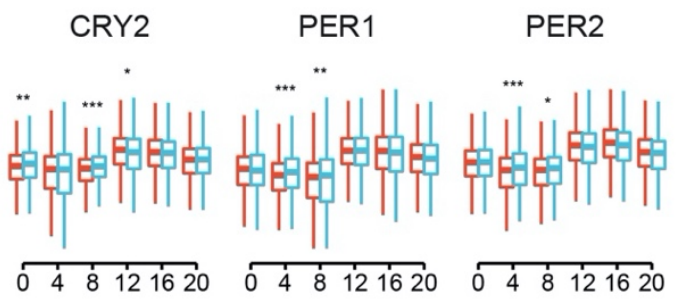

CT

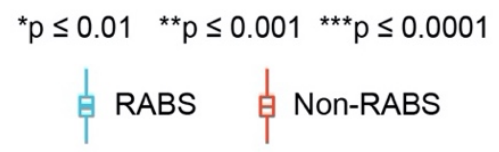

Figure 1: Repeat-derived CR binding sites display similar CR binding but less hallmarks of enhancer activity.

(A)Fraction of ChIP-seq peaks for each CR for which $>50 \%$ of the peak did (RABS) or did not (Non-RABS) map within repetitive elements.

(B) CLOCK:BMAL1 ChIP-seq peaks sorted by the nascent transcriptional output of the closest gene (Rhythmic in-phase or out-of-phase; Arr: Arrhythmic; NE: Not Expressed), classified as RABS or Non-RABS as in (A).

(C)Distribution of ChIP-seq signal in RABS ( $\mathrm{n}=1014)$ and Non-RABS $(\mathrm{n}=7197)$ BMAL1 ChIP-seq peaks across Circadian Time (CT). 

(ZT).

179 Boxplots in (C-D) show the median (center line), the $1^{\text {st }}$ and $3^{\text {rd }}$ quartiles (hinges), and $1.5 \times \mathrm{IQR}$

180 (whiskers). Significance values are from the Kruskal-Wallis test with Bonferroni-corrected Dunn 181 post-hoc comparison. 
183 (CACGTG) separated by a variable 6-7 nucleotide spacer [59]. We predicted that both RABS

184 and Non-RABS would be enriched in E-Box motifs, and found that there was indeed a strong

185 pattern of E-Box enrichment at the center of both RABS and Non-RABS peaks, but no such

186 enrichment at the center of our set of randomly selected repeats (Fig. S3A). The average distance

187 from RABS peaks and repeats to the closest E-Box was similar to that observed from Non-

188 RABS peaks, and both sets are much closer than expected by chance (Fig. S3B). Of E-Box

189 motifs associated with RABS, $82.5 \%$ fell within the boundaries of the associated repeat,

190 indicating that the repeats themselves are contributing the binding site and are directly bound by

191 CRs.

192

Because RABS are bound by CRs in a similar manner to Non-RABS, we would expect

194 that they would have similar chromatin state and regulatory potential. To interrogate chromatin

195 state and regulatory activity or CR binding sites, we used published H3K27Ac ChIP-seq, DNase-

196 seq, and Pol II ChIP-seq experiments conducted in the mouse liver in Zeitgeber time [68], which

197 is distinct from Circadian time in that animals are harvested over a light-dark cycle rather than

198 constant dark conditions. We accessed raw sequencing reads (Table 1) and processed them with

199 the software pipeline used for CR ChIP-seq data with minor adjustments (see methods).

200 Intriguingly, while the oscillatory pattern of DNase sensitivity, H3K27 acetylation, and Pol II

201 occupancy is maintained between RABS and Non-RABS, the median observed signal intensity is

202 significantly lower at RABS than at Non-RABS (Fig. 1D). We confirmed this observation by

203 visualizing individual loci as heatmaps (Fig. S2B-D). The magnitude of these differences

204 between RABS and Non-RABS is substantially greater than those observed in CR binding 
205 (compare Fig. 1C and Fig. 1D). This could be a result of differences in the unique mappability of

206 repetitive regions between experiments, but the DNase-seq, H3K27Ac ChIP-seq, and Pol II

207 ChIP-seq libraries were sequenced with longer read length than the CR ChIP-seq libraries, so the

208 unique mappability of these libraries should be better, not worse.

211 examine whether the genes proximal to E-Box motifs within RABS and Non-RABS ChIP-seq

212 peaks (two nearest genes within $1 \mathrm{Mb}$ ) are enriched for particular mouse phenotypes. GREAT

213 determines enrichment by comparing the gene associations of input sites with those of a user-

214 defined background. When we used the entire mouse genome as background, RABS and Non-

215 RABS displayed significant enrichment for similar annotations corresponding to fairly broad

216 ontologies, which are largely related to liver function or circadian phase as previously reported

217 [67] (Fig. S4A-B). We reasoned that these enrichments may be driven by the general open

218 chromatin landscape of liver cells rather than by biological processes more specifically under

219 circadian control in the liver. Indeed, when we repeated the GREAT analysis using all open

220 chromatin regions - defined by DNase accessibility at any time point — as background, a

221 different trend emerged. While Non-RABS were again enriched in phenotypes likely to be

222 related to general liver function and circadian phase (Fig. S4C), RABS were enriched in

223 categories related to pigmentation phenotypes, including abnormal digit pigmentation, non-

224 pigmented tail tip, and variable body spotting (Fig. S4D). Collectively these analyses indicate

225 that RABS and Non-RABS share similar CR occupancy and motif composition, but RABS

226 exhibit weaker signals of regulatory activity as a whole and are tied to genes whose expression

227 patterns could be related to species-specific phenotypic variation. 


\section{RABS are enriched for RSINE1 elements}

To determine if particular TEs were contributing CR RABS more often than expected

230 from their frequency in the genome, we tested for enrichment of individual mouse TE families

231 for their intersection with ChIP-seq peaks using a previously described pipeline [78]. We found

232 that several TE families were significantly enriched in each CR dataset, but after imposing a

233 cutoff of $>50$ observed bound copies per family, only a single family, RSINE1, stood out across

234 5/6 ChIP-seq datasets (Fig. 2A). While RSINE1 did not meet our threshold of 50 elements bound

235 in the PER1 ChIP-seq data with 43 unique elements bound, enrichment of RSINE1 was still

236 statistically significant. For each set of CR binding sites there were between 43 and 242

237 RSINE1-derived RABS, representing 3.2 to 5.4-fold enrichment over expectation (Fig. 2B).

238 Importantly, other related B4 SINE families including B4, B4A, and ID_B1 were only

239 significantly enriched for binding of 1-3 of the CRs and no consistent trend was apparent. In

240 total, 328 unique RSINE1 elements in the genome were bound by at least one CR. Application of

241 this method to CLOCK:BMAL1 peaks sorted by the nascent transcriptional output of the nearest

242 gene [63] revealed similar levels of RSINE1 enrichment among 'arrhythmic' and 'not expressed'

243 classes of binding sites, but no significant increase in RSINE1 elements in 'rhythmic in-phase'

244 transcriptionally cycling binding sites (Fig. 2C). No RSINE1 elements were associated with

245 'rhythmic out-of-phase' transcriptionally associated binding sites. This corroborates our previous

246 speculation that TE-derived CR binding sites are less likely to have biological relevance than

247 non-RABS CR binding sites.

To investigate why some RSINE1 elements are bound by CRs while others are not 250 despite their identical sequence upon insertion, we first compared temporal CR binding patterns, 
251 chromatin landscape, and Pol II occupancy between RSINE1 elements predicted to be bound by

252 CRs and those apparently unbound but still residing in open chromatin (OC; Unbound). We

253 reasoned that RSINE1 elements in open chromatin regions might be more informative for

254 comparison than random elements, though by doing so we are more likely to introduce false

255 negatives in our unbound set. We found that there were strong oscillatory patterns of CR binding

256 enriched at these bound RSINE1s, and while the pattern of enrichment on the unbound fraction

257 was also oscillatory, it was weaker and more diffuse (Fig. 3A). We verified that this is only true

258 of CR-unbound OC RSINE1s by inspecting heatmaps of unbound RSINE1s that do not reside in

259 open chromatin and observed no detectable oscillatory CR ChIP-seq signal on these elements

260 (Fig. S5). While this was somewhat surprising, we reasoned it could occur if these unbound OC

261 RSINE1s were bound by other TFs themselves under circadian regulation or resided in genomic

262 locale broadly regulated by CRs.

263

265 hypersensitivity (Fig. 3B), H3K27 acetylation (Fig. 3C), and Pol II occupancy (Fig. 3D)

266 characteristic of active circadian enhancers. These patterns were rarely apparent, diffuse, and off-

267 center relative to RSINE1 when examining unbound OC RSINE1s (Fig. 3B-D). While we

268 expected that these OC RSINE1s would show DNase hypersensitivity—we selected this set

269 precisely for this property - the oscillatory nature of the accessibility was surprising. We

270 speculate this is driven by bound RSINE1s which did not meet the stringent $50 \%$ overlap

271 criterion used in this analysis, and as such, our set of bound RSINE1s is likely an underestimate.

272 Indeed, when we intersected our randomly selected set of unbound OC RSINE1 elements with

273 BMAL1 ChIP-seq peaks, we found that 20/328 (6\%) of these elements would have been 
bioRxiv preprint doi: https://doi.org/10.1101/2020.11.09.375469; this version posted November 10, 2020. The copyright holder for this preprint (which was not certified by peer review) is the author/funder, who has granted bioRxiv a license to display the preprint in perpetuity. It is made available under aCC-BY 4.0 International license.

274 classified as RABS if the overlap criterion was $25 \%$ instead of $50 \%$. Taken together these results

275 indicate that RSINE1 elements contribute a disproportionate fraction of CR TFBSs in the mouse

276 liver, and that CR-bound RSINE1 elements display hallmarks of active regulatory elements. 

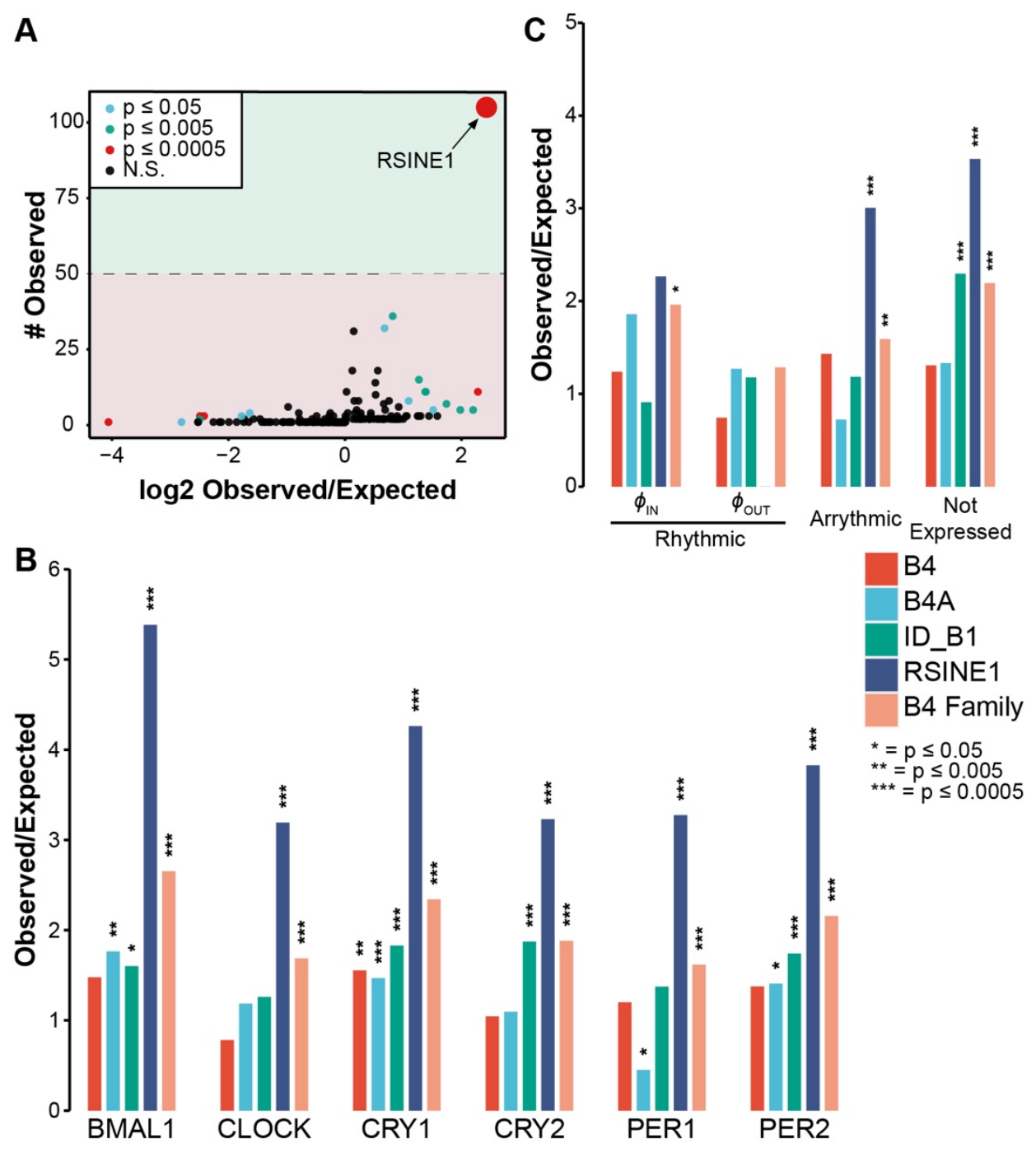

Figure 2: RSINE1 elements are overrepresented in RABS.

(A) All TEs detected in BMAL1 ChIP-seq peaks, plotted by the number of elements observed compared to the ratio of observed to expected occurrences for that particular TE. Expected values were calculated by bootstrapping 1000 times (see methods). The dashed line denotes our cutoff of $>50$ elements observed imposed to filter false positives.

(B) Ratio of observed to expected occurrences, as in (A), for individual members of the B4 family and for the entire B4 family, in sets of RABS associated with each CR. 
bioRxiv preprint doi: https://doi.org/10.1101/2020.11.09.375469; this version posted November 10, 2020. The copyright holder for this preprint (which was not certified by peer review) is the author/funder, who has granted bioRxiv a license to display the preprint in perpetuity. It is made available under aCC-BY 4.0 International license.

(C) As in (B), but enrichment of TEs in high confidence CLOCK:BMAL1 binding sites categorized by their nascent transcriptional output.

288 All p-values were obtained using a two-sided binomial test. 


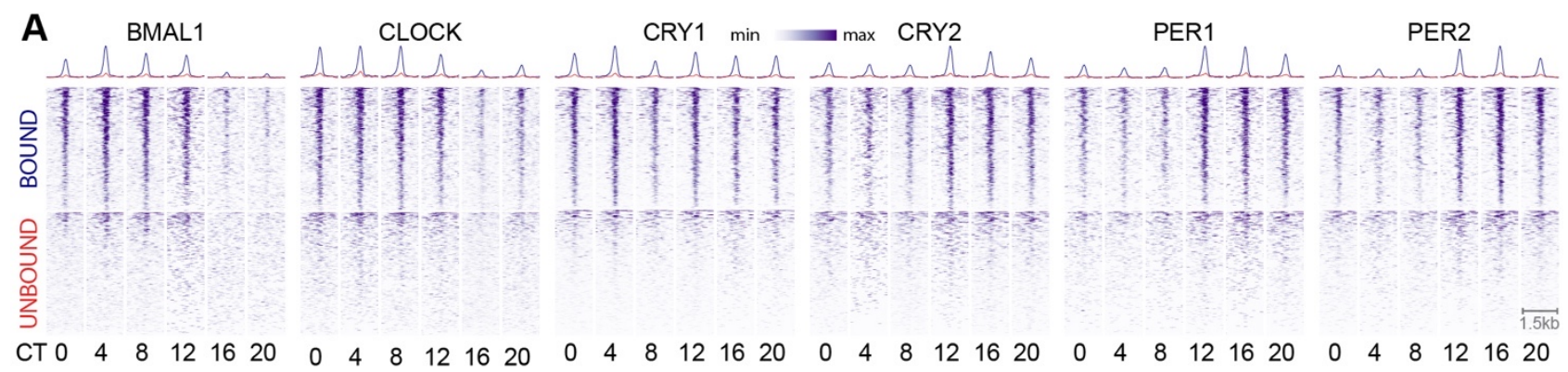

B

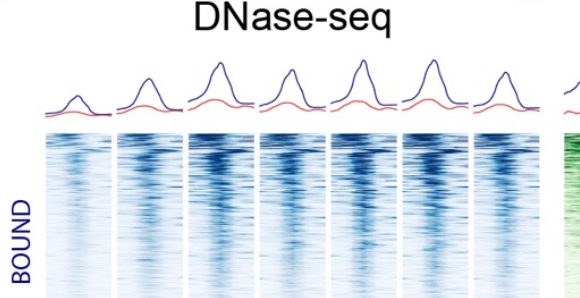

C H3K27Ac ChIP-seq

D
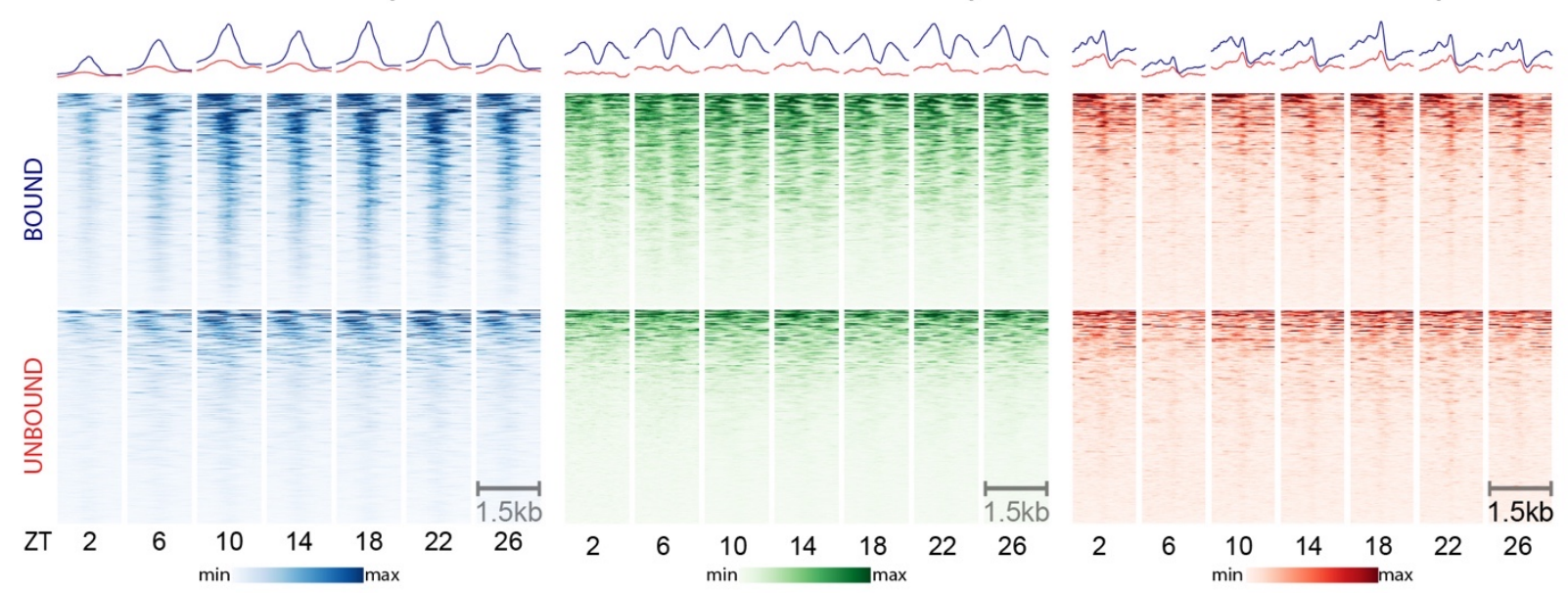

Figure 3: Differential CR occupancy, chromatin landscape, and Pol II occupancy of bound

(A) ChIP-seq signal for each CR across circadian time (CT), centered at CR associated RSINE1 elements ( $\mathrm{n}=328$, top) and a randomly selected set of 328 RSINE1 elements which fall within open chromatin regions as defined by DNase-seq but are not associated with CR binding ( $\mathrm{n}=328$, bottom). Heatmaps are centered at the middle of each element in positive orientation, extend $750 \mathrm{bp}$ in each direction and are sorted by mean signal intensity across all rows for a given CR.

(B) DNase-seq signal over Zeitgeber time (ZT). Regions, sorting, and centering as in (A). in (A). 
bioRxiv preprint doi: https://doi.org/10.1101/2020.11.09.375469; this version posted November 10, 2020. The copyright holder for this preprint (which was not certified by peer review) is the author/funder, who has granted bioRxiv a license to display the preprint in perpetuity. It is made available under aCC-BY 4.0 International license.

$301 \quad$ (D) Pol II ChIP-seq signal over Zeitgeber time (ZT) Regions, sorting, and centering as in (A).

302 In all panels color scaling is relative to min-max signal within each block of heatmaps. Trace

303 above heatmaps is the average signal in each class. 
CR binding of RSINE1s is explained by sequence motif composition.

We reasoned that the ancestral (consensus) sequence of RSINE1 might explain its in the mouse genome are the B4 and B4A families $[40,79,80]$. While some other members of the

309 B4 superfamily are significantly enriched to varying degrees in CR binding sites, none show

310 levels of enrichment comparable to that of RSINE1 (Fig. 2B). Thus, we hypothesized that

311 RSINE1 may have unique sequence motifs that predispose these elements to recruit CRs. To test 312 this idea, we compared the consensus sequences of RSINE1, B4, and B4A obtained from 313 Repbase [74] for the presence of E-box motifs (CACGTG), which are recognized by CLOCK 314 and BMAL1, as well as RORE motifs (RGGTA), which recruit nuclear receptors (NRs). NRs are 315 known to be important co-regulators of circadian gene expression in the liver and include ROR $\alpha$

316 [81], REV-ERBs [82], and LXR/RXR [83], which have well characterized roles in liver 317 metabolism [71] and circadian transcriptional regulation [57,66]. We also searched for D-box 318 motifs, which are bound by TEF, HLF, and DBP; three additional circadian TFs that orchestrate 319 rhythmic transcription in different phases [57]. sequence (Fig. 4A). All of the E-box motifs and two of the three RORE motifs present were not

323 perfect matches to the empirically determined, optimal motifs for binding of CRs and NRs.

324 Hereafter we call these imperfect motifs "proto-motifs". A pair of closely spaced (6-7 nt) E-Box 325 motifs (CACGTG) is the optimal CLOCK:BMAL1 heterodimer binding site [59]. We observed 326 one E-Box that is a single nucleotide change (CACATG to CACGTG; E-Box 1; Fig 4A) away 
327 from the optimal sequence in the center of the RSINE1 consensus sequence; however this motif

328 is less likely to contribute significant regulatory activity as it is not a tandem motif, and similar

329 sequences were present in B4 and B4A consensus sequences. Near the end of the RSINE1

330 consensus sequence, there were two E-Box motifs separated by 6 nt that are each a single

331 nucleotide change away from the optimal sequence (CACGCG to CACGTG; E-Box 2 \& 3; Fig.

332 4A). These two ancestral motifs each would require a single $\mathrm{C}$ to $\mathrm{T}$ mutation $-\mathrm{a}$ common

333 deamination mutation in methylated DNA - to match the preferred CLOCK:BMAL1 binding

334 site. The first RORE motif in the RSINE1 consensus is a perfect antisense motif and is closely

335 followed by a sense motif that is two nucleotides away from an optimal RORE motif. However,

336 these motifs are also present in the B4 and B4A consensus sequences. Together, these

337 observations led us to conclude that E-Boxes $2 \& 3$ are the unique property of RSINE1 which

338 most likely predisposed it to circadian regulatory activity.

We next sought to understand why a subset of RSINE1 copies in the genome are bound

341 by CRs while most are not. We searched for motifs enriched in CR-bound RSINE1 elements

342 with respect to OC RSINE1s and all RSINE1s. Discriminative unbiased motif discovery returned

343 CACGTG and AGGKCA as enriched in bound RSINE1 elements with respect to unbound

344 RSINE1 elements. Strikingly, these motifs correspond to E-Box and RORE elements

345 respectively (Fig. 4B). To further explore the motif composition of these elements, we plotted

346 profiles of E-Box and RORE motif occurrence weighted by motif confidence over $200 \mathrm{bp}$

347 regions around the center of bound and OC unbound RSINE1s (Fig. 4C). Bound and unbound

348 fractions both displayed characteristic patterns of tandem E-Box and RORE motifs, but few D-

349 Box motifs. Notably, the summary plot revealed that while the spatial pattern is similar, the 
350 bound fraction has higher signal strength, which indicated that bound RSINE1 elements more

351 frequently contain motifs that more closely match the optimal sequence than unbound OC

352 RSINE1 elements.

353

We next examined the density distribution of distance from each optimal E-Box motif and the next closest E-box motif in order to identify characteristic spacing, which might better explain the difference between bound and unbound RSINE1 elements. Strikingly, the bound

357 class had a strong enrichment for an E-Box $\sim 12$ bp from the nearest optimal E-Box, which was 358 not present in unbound OC RSINE1 elements (Fig. 4D) and coincides with the pair of proto359 motifs at the 3' end of the consensus (Fig. 4A). When we examined the distance from RORE to

360 RORE, we observed enrichment of closely adjacent motifs in both classes of RSINE1 elements

361 (Fig. 4E). These results are consistent with the spacing of proto-motifs in the RSINE1 consensus

362 and support the model that the bound class of RSINE1s gained their regulatory activity after 363 transposition via maturation of imperfect CR/NR binding sites.

To verify that identified RORE elements are actually bound by NRs in the mouse liver, 366 we turned to published ChIP-seq data for REV-ERBs [71], RXR, LXR, PPAR $\alpha$ [70], and ROR $\alpha$

367 [69] (Table 1). These datasets revealed that bound RSINE1 elements were occupied by the

368 aforementioned NRs, and that different NRs were bound simultaneously at the same RSINE1

369 elements, indicating that different NRs and/or CRs could be binding to these elements

370 cooperatively. A randomly selected subset of unbound RSINE1s in open chromatin also

371 displayed some NR binding but not to the degree of CR bound RSINE1s (Fig. 4F). We reasoned 372 that because NRs are liver-specific circadian co-regulators, the interplay of these CR/NR binding 
373 sites in the RSINE1 consensus may have given rise to tissue-specific regulatory elements. We

374 then leveraged a recent analysis of tissue specific BMAL1 binding sites using ChIP-seq in the

375 mouse liver, heart, and kidney [72], and found that while the fraction of peaks overlapping with

376 repetitive elements was relatively consistent, the fraction overlapping with RSINE1 elements

377 was highest in the liver and markedly lower in other tissues (Fig. 4G). These results suggest that

378 the majority of RSINE1-derived CR binding sites are liver-specific.

To rule out the possibility that the E-Box and RORE motifs enriched in bound RSINE1s

381 could have emerged from the expansion of a slightly different progenitor containing pre-existing

382 optimal motifs but forming a distinct RSINE1 subfamily, we aligned all CR-bound RSINE1

383 elements located in open chromatin with 1000 randomly selected RSINE1 copies and performed

384 a phylogenetic analysis, the resulting tree had star-like topology with no subfamily structure

385 coinciding with the two categories (Fig. 4H). This confirms that both bound and unbound

386 RSINE1 elements descend from the same ancestral sequence which amplified rapidly throughout

387 the genome. These results led us to conclude that RSINE1 spread imperfect CR/NR binding sites

388 throughout the mouse genome, providing fodder for the evolution of new circadian enhancers

389 rather than spreading 'ready-made' enhancer modules. 


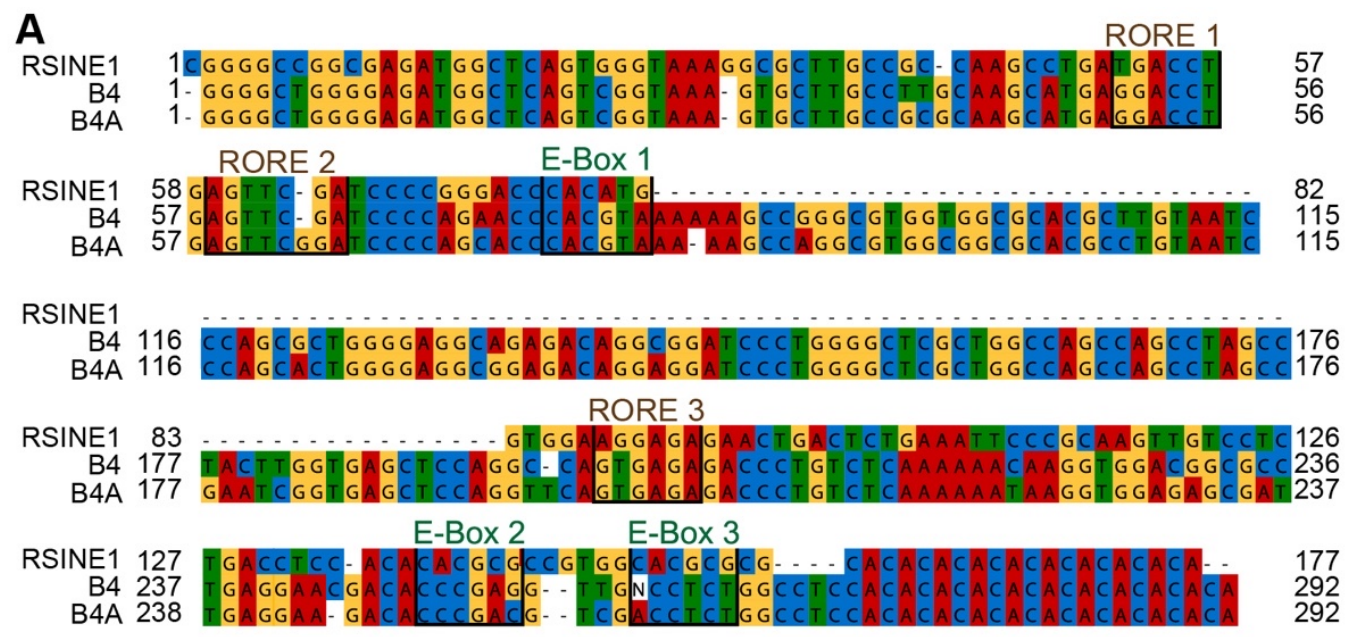

B

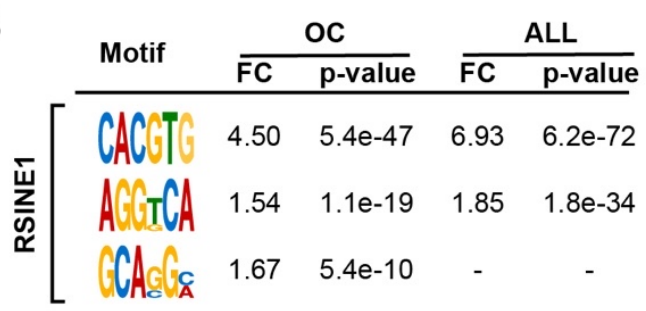

C

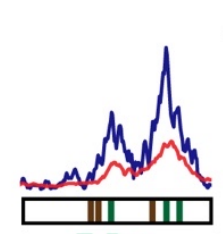

E-Box

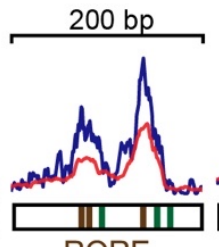

RORE

D

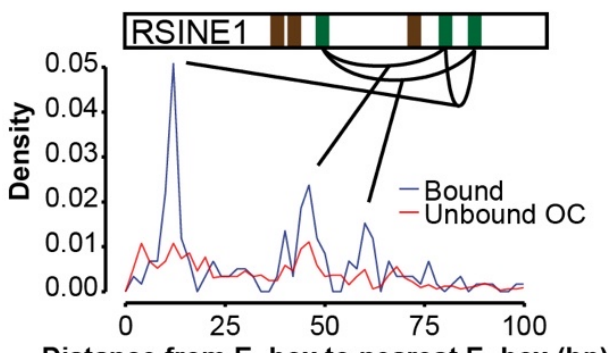

Distance from E-box to nearest E-box (bp)

E

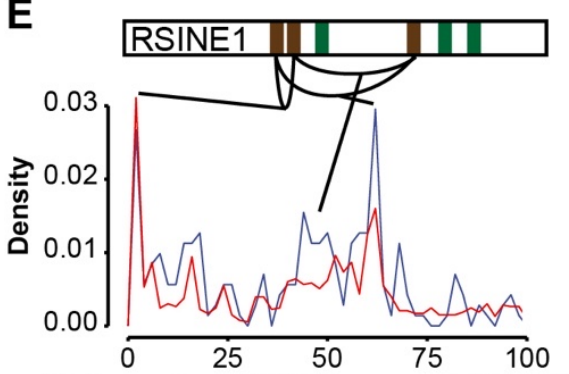

Bound

Unbound OC

$\mathbf{F}$

Bound

RSINE1

Unbound

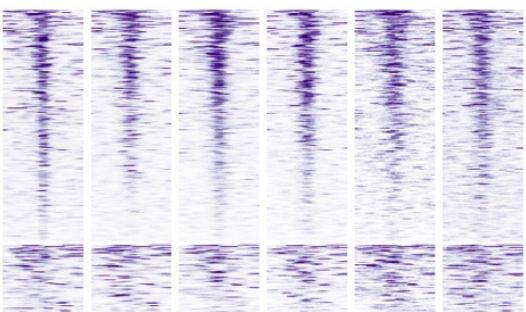

$\max$

OC

RSINE1

$\min$
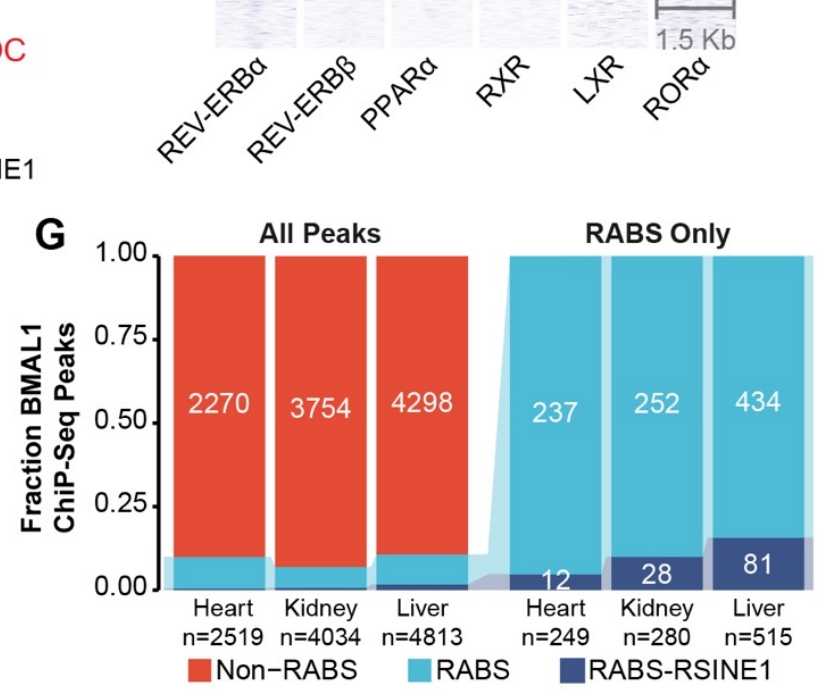

H

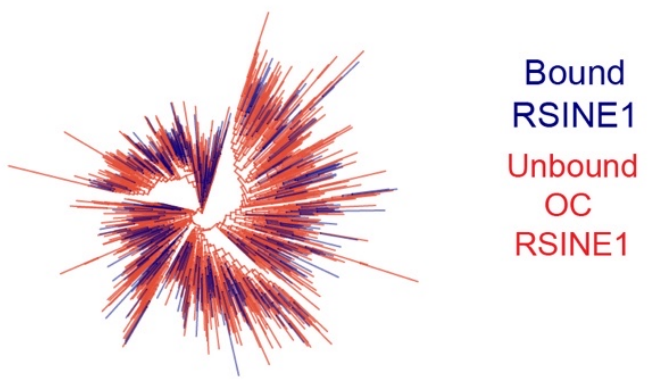




\section{Figure 4: RSINE1 elements contain unique motif composition.}

393 (A) Multiple sequence alignment of B4 family SINES. RORE and E-Box pre-motifs are highlighted.

(B) Motifs enriched in bound RSINE1 elements relative to unbound RSINE1 elements which reside in open chromatin (OC), or relative to all RSINE1 elements (ALL). The motif enrichment calculation was also performed using $500 \mathrm{bp}$ of flanking sequences. within bound (blue line; $\mathrm{n}=328$ ) and unbound OC (red line; $\mathrm{n}=4279$ ) classes of RSINE1 elements. Plots are centered on RSINE1s and extended $100 \mathrm{bp}$ in either direction. RSINE1 elements ( $\mathrm{n}=328)$ and unbound open chromatin RSINE1 elements $(\mathrm{n}=4279)$. $(n=328)$ or a randomly selected subset of unbound OC RSINE1 elements $(n=328)$. Sort order is maintained across columns; color is scaled relative to min-to-max for each individual factor (column).

(G)Fraction of BMAL1 ChIP-seq peaks in the mouse heart, kidney, and liver which are 
415 Circadian enhancer evolution from RSINE1 elements is context-dependent and lineage416 specific.

417 We next asked if the genomic location where RSINE1 inserted influences their

418 propensity to act as circadian regulatory elements. More specifically, we asked whether the

419 presence of an existing CR-bound sequence might favor the emergence of CR binding at

420 RSINE1 elements. We determined the distance from each bound RSINE1 to the nearest non-

421 repeat derived CR binding site and compared this to the expected distance using the same

422 calculation but using all genomic copies of RSINE1. Bound RSINE1s are significantly closer to

423 Non-RABS (median $\approx 8 \mathrm{~kb}$ ) than expected given the genomic distribution of all RSINE1

424 elements (median $\approx 65 \mathrm{~kb}$; Fig. 5A). To determine whether these Non-RABS existed prior to

425 RSINE1 insertion and thus shaped the evolutionary trajectory of RSINE1, we then queried

426 whether Non-RABS CR binding sites have deeper phylogenetic roots than those in associated

427 with repetitive elements by determining the fraction of regions for which an orthologous region

428 could be identified in the rat and human genomes using liftOver [84]. We found that $\sim 46 \%$ of the

429 Non-RABS ChIP-seq peaks could be traced to orthologous regions in the human genome, and $430 \sim 74 \%$ to the rat genome (Fig. 5B). This percentage is higher than the overall percentage of the

431 mouse genome ( $40 \%)$ that can be aligned confidently at the nucleotide level with the human

432 genome [40], indicating that Non-RABS tend to be deeply conserved. Consistent with RSINE1's

433 murine specificity, $\sim 60 \%$ of bound RSINE1 elements could be traced to an orthologous region in

434 the rat genome, but virtually none $(0.3 \%)$ were detected in the human genome (Fig. $5 \mathrm{C})$. Thus, in 435 general, RSINE1 derived CR binding sites are evolutionarily younger than Non-RABS and must 436 have inserted nearby existing CR binding sites prior to their maturation into circadian enhancers.

437 Given our previous finding that RSINE1 elements contained proto-motifs prior to their 
438 expansion, this result suggests that RSINE1 elements preferentially matured into optimal

439 circadian regulatory elements after insertion nearby existing circadian regulatory sequences.

441 RSINE1 elements appear to have distributed imperfect CR/NR binding sites prior to the

442 divergence of the mouse and rat lineages, and we hypothesized that they occasionally matured

443 into lineage- or tissue-specific circadian enhancers. To test this, we queried syntenic orthologs of

444 mouse bound and unbound OC RSINE1 elements in the rat and performed motif discovery. This

445 revealed that most elements which have developed perfect E-Box motifs (CACGTG) in the

446 mouse have not developed the same motif in the rat (Fig. 6C). This indicates that bound RSINE1

447 elements in the mouse represent a subclass of RSINE1 elements which gained CR binding after

448 the divergence of mouse and rat and thus are lineage specific CR binding sites. Together these

449 findings show that the evolution of circadian regulatory elements from the proto-motif content of

450 RSINE1 is a process which is context-dependent and lineage-specific. 


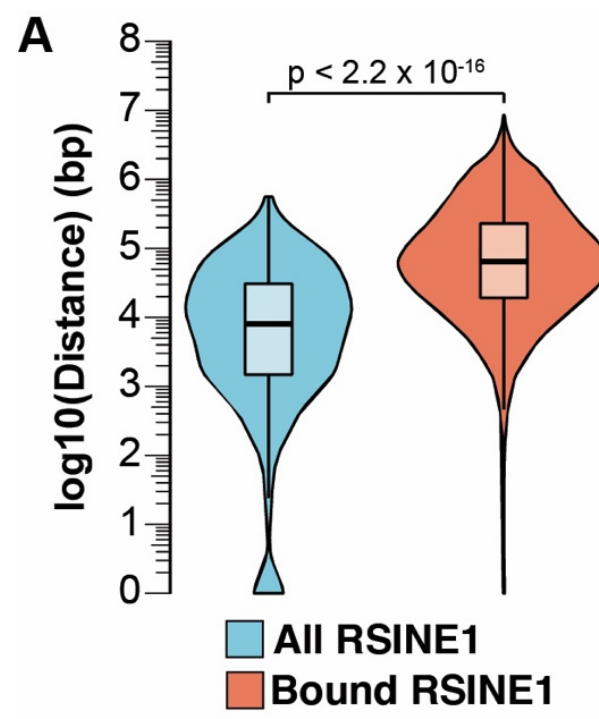

B

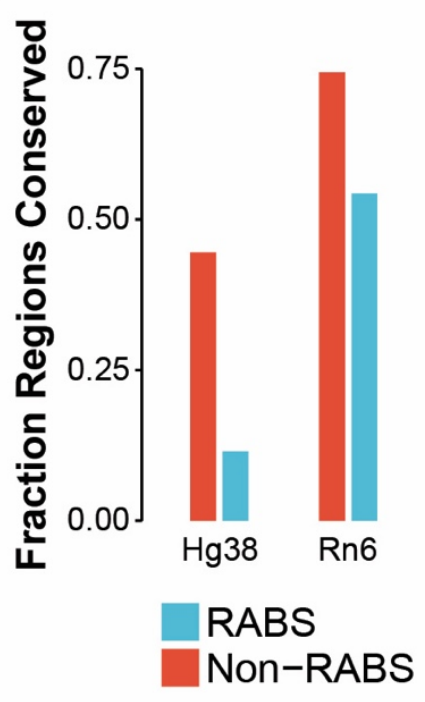

C

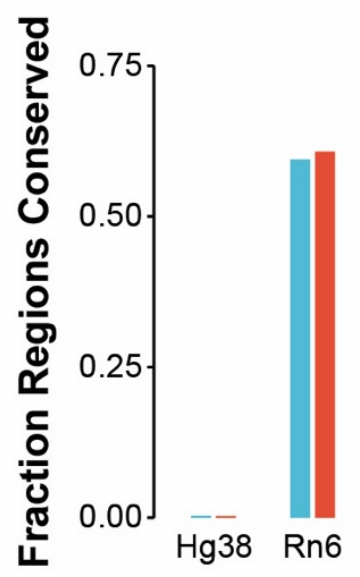

D

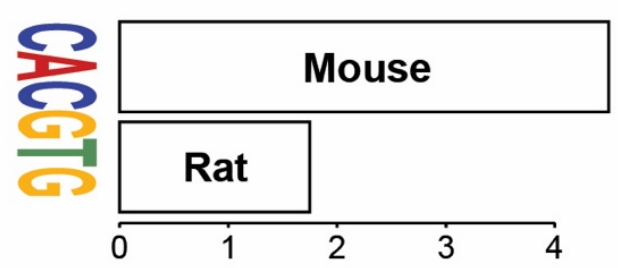

Fold Change Motif Abundance Bound RSINE1 vs. Unbound OC RSINE1

452 . Figure 5: Evolution of CR binding sites from RSINE1 is context dependent.

453 (A)Distribution of distance from each CR bound $(n=328)$ or all $(n=115,806)$ RSINE1

454 elements to the nearest non-repetitive CR binding site (Non-RABS). Boxplots show the

455 median (center line), the $1^{\text {st }}$ and $3^{\text {rd }}$ quartiles (hinges), and $1.5 \times$ IQR (whiskers). Statistical

456 testing was done using the Mann-Whitney/Wilcoxon rank sum test.

457 (B) Fraction of Non-RABS and RABS CR bindings sites that can be traced to the human or $458 \quad$ rat genomes using liftOver. 
459 (C)Fraction of CR bound and unbound RSINE1 elements that can be traced to the human or $460 \quad$ rat genomes using liftOver.

461 (D)Fold change CACGTG motifs detected in bound RSINE1 elements compared to unbound 462 OC RSINE1 elements in the mouse as well as in syntenic orthologs in the rat. 
464 A minority of RSINE1 elements have matured into circadian enhancers.

The above data indicate that RSINE1 elements are poised for maturation into circadian

466 regulatory elements. We next searched for evidence for circadian enhancer activity of RSINE1

467 elements. A published study [69] used GRO-seq (Table 1) to measure nascent transcription over

468 circadian time in the mouse liver and identified enhancer RNAs (eRNAs) that transcriptionally

469 oscillate. We intersected our set of CR-bound RSINE1 elements with the coordinates of these

470 oscillating eRNAs and identified 37 RSINE1 elements (11.3\% of bound RSINE1s) for which

$47150 \%$ of the RSINE1 element falls within the coordinates of an oscillatory eRNA. One such

472 example is an RSINE1 element that falls within the last intron of Paipl and is $\sim 6 \mathrm{~kb}$ upstream of

473 the start of 4833420 G17Rik, a cDNA with no identified function, which is in turn $\sim 2 \mathrm{~kb}$ upstream

474 of the TSS of Tmem267, a gene with a CR-bound promoter (Fig. S6A). Furthermore, an

475 independent study [63] identified 4833420G17Rik as transcriptionally oscillating using Nascent-

476 seq, and the RSINE1 element also overlapped with a high confidence BMAL1:CLOCK binding

477 site designated as associated with rhythmic in phase transcription. Upon further inspection, we

478 observed that the RSINE1 element had a characteristic pattern of oscillating CR binding and

479 chromatin accessibility and was bound by NRs (Fig. S6A). When we examined the sequence

480 composition of this RSINE1, we observed two E-Box motifs at the end of the element and a

481 RORE motif in the center. A D-Box motif was also present slightly upstream of the element,

482 indicating that this RSINE1 could have matured into a bona fide circadian enhancer by virtue of

483 the genomic context it inserted into. Another RSINE1 that falls within an oscillatory eRNA but

484 does not have a D-Box in the flanking sequence lies immediately upstream of the promoter of

485 Aaedl (Fig. S6B). 
We next used in vitro luciferase assays in Hepa 1-6 cells, a mouse hepatoma-derived cell

488 line, to measure the enhancer activity of selected RSINE1 sequences. Importantly we verified

489 that CLOCK and BMAL1 are constitutively expressed in this cell type using public RNA-seq

490 data (Table 1). We initially compared the consensus sequence from Repbase [74] to an "in silico

491 evolved" sequence that we subjected to a total of 7 nucleotide substitutions to perfect the

492 degenerate motifs in the RSINE1 consensus. We observed that the consensus alone had moderate

493 enhancer activity, and the "evolved" version's activity was significantly boosted (Fig. 7A). Next,

494 we examined the genomic RSINE1 elements described above (Tmem267 and Aaed1). For each,

495 we tested the regulatory activity of the RSINE1 element alone, the RSINE1 with 100 bp flanking

496 sequences, and a proxy for the ancestral locus where the RSINE1 was removed leaving only the

497 flanking sequences joined at the insertion site. In both cases, we found that the RSINE1 element

498 alone had the highest activity, and that this activity was slightly lower when the flanking

499 sequences were included (Fig. 6A). Removing the RSINE1 had no effect on the activity of the

500 flanking sequences in the Aaed1 reporter, but completely abolished luciferase signal of the

501 Tmem 267 reporter. This pattern was consistent with the presence of a D-Box motif in the

502 upstream flank of the Tmem267, which is known to be bound by the transcriptional repressor

503 E4BP3 (also called NFIL3) as part of a secondary circadian transcriptional feedback loop with

504 opposing phase to the classic CLOCK:BMAL feedback loop [85]. The most likely explanation

505 for the phenomenon observed at the Aaed1 RSINE1 is that the flanking sequence contains both

506 activating and repressive TFBSs which in balance yields mild basal enhancer activity, and while

507 the RSINE1 contains stronger regulatory activity on its own, its presence in the flanking

508 sequence does not disrupt this balance in a manner sufficient to alter enhancer activity. These

509 assays illustrate how RSINE1 has shaped the circadian regulatory landscape of the murine 
bioRxiv preprint doi: https://doi.org/10.1101/2020.11.09.375469; this version posted November 10, 2020. The copyright holder for this preprint (which was not certified by peer review) is the author/funder, who has granted bioRxiv a license to display the preprint in perpetuity. It is made available under aCC-BY 4.0 International license.

510 lineage (Fig. 8) and highlight how the genomic context of each insertion of an identical family of

511 repetitive elements dictates which elements gain TF binding and regulatory activity each in

512 unique ways. 


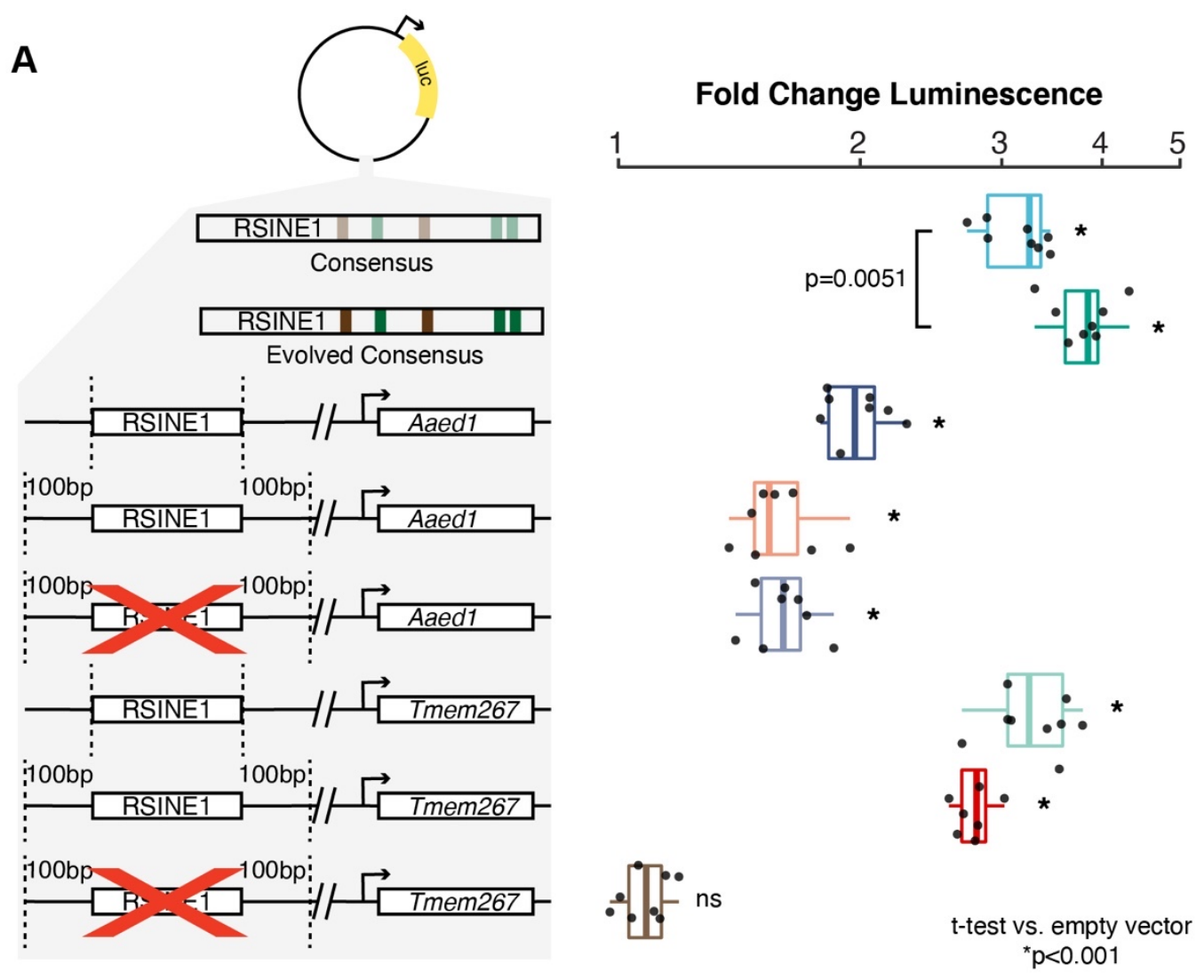

B

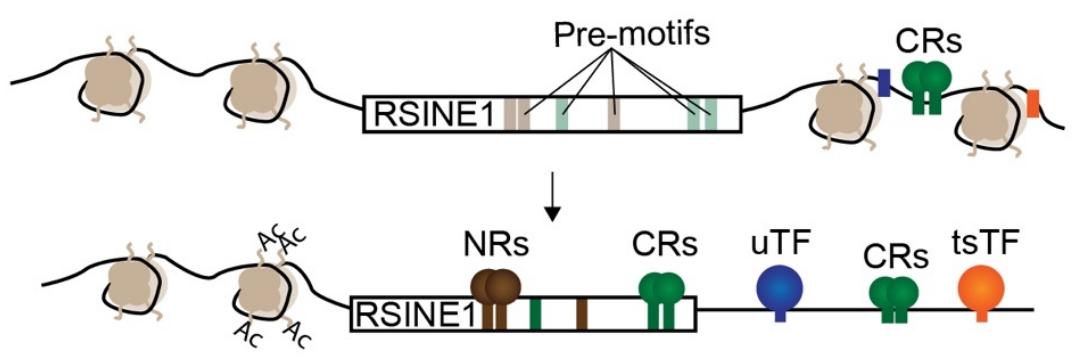

514 Figure 6: RSINE1 elements have enhancer activity in vitro.

515 (A) Fold change luminescence when compared to an empty vector control after

516 normalization to Renilla luciferase signal of enhancer reporter plasmids with various

517 RSINE1-derived sequences inserted downstream of the luciferase gene. Included are:

518 RSINE1 consensus as is or with "evolved" E-Box and RORE motifs at highlighted

519 locations (all motifs were changed to the canonical motif with no other changes), an

520 RSINE1 element upstream of the promoter of Aaedl (See Fig. S6B), and an RSINE1 
upstream of the promoter of 4833420G17Rik and Tmem267 (See Fig. S6A). reconstruction of the ancestral state. Experiments are representative of two biological transfection replicates with four technical replicates each. Significance was assessed using pairwise two-tailed t-tests compared to the empty vector. murine lineage by distributing imperfect $\mathrm{CR} / \mathrm{NR}$ binding sites with moderate elements. 


\section{Discussion}

In this study, we show that a subset of CR binding sites in the mouse liver map within

534 repetitive elements. This class had patterns of oscillatory CR binding that were indistinguishable

535 from their non-repeat derived binding sites, but characteristics consistent with their function as

536 bona fide circadian enhancers were weaker than those observed for non-repeat derived binding

537 sites. This is in line with recent observations that only a small subset of TEs which display

538 enhancer marks in mouse embryonic stem cells trigger gene expression changes when perturbed

539 [86]. Furthermore, GO analysis of genes associated with TE-derived CR binding events show

540 enrichment for genes linked to morphological phenotypes, including non-pigmented tail tip and

541 variable body spotting, which are likely to be intra-specifically variable. We speculate that these

542 associations reflect cis-regulatory connections that are evolutionarily young and might drive

543 phenotypic plasticity akin to what is seen at the Agouti gene in mice, where the differential

544 regulatory activity of IAP individuals lead to coat color variation [87-89]. Further study is

545 needed to evaluate whether CR-bound TEs have directly contributed to changes in circadian

546 gene expression with possible phenotypic consequences.

\section{Seeding and maturation of proto-motifs by transposition.}

549 In this study, we examined the properties that predispose one family of TEs, RSINE1, to

550 be bound by CRs and to acquire the biochemical hallmarks of circadian enhancers. We focused

551 on the RSINE1 family because it was the only TE family in the mouse genome that was

552 significantly overrepresented for CR-binding events across all ChIP-seq datasets examined,

553 suggesting that this family was in some way predisposed to be bound by CR. Furthermore, we 
554 observed that many of the CR-bound RSINE1 were also bound by NRs which are important

555 regulators of circadian gene expression specifically in the liver [90,91]. In addition to being

556 bound by CRs and NRs, a small subset of these RSINE1 elements displayed features

557 characteristic of circadian enhancers, including oscillatory H3K27 acetylation, DNase

558 accessibility, and nascent transcription of eRNAs. We found that the recurrent binding of CRs

559 and NRs to RSINE1 elements can be explained in part by the presence of sequence motifs in

560 their ancestral sequence that are close, but imperfect match to the E-Box and RORE motifs that

561 recruit $\mathrm{CR}$ and NR, respectively. In particular, a feature unique to RSINE1 when compared to

562 other closely related SINE families, is a pair of imperfect E-Box motifs at the 3' end of the

563 RSINE1 consensus sequence. Thus, unlike other cases of TE families enriched for TF binding

564 events [20,45,48,50,51,92,93], RSINE1 did not introduce perfect TFBSs upon integration, but

565 proto-motifs that repeatedly matured into optimal TFBSs within individual TE copies after their

566 genomic integration.

Our analysis indicates that recurrent C-to-T mutations within the two proto E-boxes

569 located at the 3' end of RSINE1 represent the primary mutational path by which these elements

570 acquired canonical CR binding sites. Interestingly, the C-to-T transition required within each of

571 the proto-motif is at a $\mathrm{CpG}$ site (CACGCG-> CACG $\underline{\mathrm{CG}}$ ), which in mammalian genomes is $\sim 10$

572 times more likely to occur compared to mutations at non-CpG sites because of spontaneous

573 deamination of methylated cytosine [94-96]. Because SINEs are heavily methylated in the

574 mouse germline [97-99], it is likely that deamination at methylated CpG sites accelerated the

575 maturation of the proto E-boxes residing within RSINE1 elements. The birth of TFBSs via CpG

576 deamination in TEs has been observed previously in human Alu SINEs, which harbor proto- 
577 motifs for p53, PAX-6, and Myc that frequently mutate to introduce new binding sites for these

578 factors $[100,101]$. Interestingly, Myc also binds an E-Box motif, though the proto-motif in the

579 Alu consensus is CGCGCG, and thus requires two C-to-T transitions to mature into the

580 canonical CACGTG motif. Our findings bolster the proposal that CpG deamination-mediated

581 evolution of TFBSs from TE substrates is a pervasive force driving the emergence of new

582 regulatory elements $[100,101]$. This may be especially common for motifs such as the E-box for

583 which many possible configurations of proto-motifs can generate a canonical motif (CACGTG)

584 via $\mathrm{CpG}$ deamination. This is notable because the E-box is the binding motif of multiple TFs

585 with diverse physiological or developmental functions, including CLOCK:BMAL1, MYC, and

586 MYOD.

587

Genomic environment of TE insertions influences their regulatory trajectory.

Our study of RSINE1 also underscores the role of the cis-regulatory context surrounding

590 a TE insertion site in modulating the cis-regulatory potential of that TE. Our analyses indicate

591 that RSINE1 elements that inserted nearby existing CR binding sites were predisposed to acquire

592 CR-binding activity upon maturation of their E-Box proto-motifs. This observation suggests a

593 scenario whereby the insertion of RSINE1 near pre-existing CR binding sites determined, at least

594 in part, whether they would become bound by CRs. In other words, the genomic regions where

595 CRs were already binding at the time of RSINE1 amplification represented a favorable

596 environment for the recruitment of CR to RSINE1 inserted in these regions. It is also possible

597 that RSINE1s were only permitted to develop bona fide CR/NR binding sites when their

598 insertion location allowed this gain of activity to fine-tune, replace, or become redundant with

599 existing circadian regulatory elements, while the introduction of such regulatory activity at other 
600 genomic locations might have been deleterious. Our reporter assays are consistent with the

601 notion that the genomic sequences flanking CR bound RSINE1 elements temper their cis-

602 regulatory activity. This is in line with the idea that RSINE1 elements that were permitted to

603 mature into CR enhancers did so preferentially when inserted in genomic regions that buffered

604 the deleterious potential of aberrant circadian regulatory activity.

605

607 located nearby existing CR BS is reminiscent of a previously proposed model dubbed "epistatic 608 capture" [102]. The authors reported that the combination of a MER20 and MER39 element

609 form the enhancer and promoter of decidual prolactin in humans. By tracing the evolutionary

610 history of these elements, they demonstrated that the MER39 element inserted nearby the

611 preexisting MER20 element, after which 7 nucleotide substitutions added 4 TFBSs which

612 together drove elevated expression of prolactin. The authors argue that the presence of nearby

613 TFBSs caused the stabilization of motifs and maturation of proto-motifs residing within the

614 MER39 element [102]. Our findings for RSINE1 are in agreement with this model and suggest

615 that the cis-regulatory landscape surrounding the insertion site of a TE profoundly influences its

616 mutational path and cis-regulatory trajectory.

619 turnover of TFBSs and cis-regulatory elements during evolution. Our data for RSINE1 suggest

620 that RSINE1 are more likely to acquire a CR BS when they inserted near an existing CR BS.

621 This process, in turn, would lead to redundancy in CR-binding, which in some cases might lead

622 to the mutational decay of the ancestral binding site, or be preserved by natural selection as a 
623 'buffer' against harmful cis-regulatory variation [103]. Choudhary and colleagues recently

624 reported an elegant demonstration of redundant TFBSs introduced by transposition [104]. The

625 authors show that TE-derived CTCF sites inserted nearby existing CTCF sites in human and

626 mouse reinforce and maintain conserved higher order chromatin structure by conferring

627 redundancy to conserved CTCF sites. The motif gain we see in RSINE1 insertions nearby

628 existing CR BS could represent binding site turnover events. It is difficult to rigorously assess

629 this without information regarding BMAL1 binding in the liver of other species, but this is an

630 idea worthy of further investigation.

631

\section{Conclusions.}

633 In summary, we propose that evolution of circadian regulatory elements from RSINE1

634 has followed this trajectory: (a) RSINE1 expanded in a single burst of rapid amplification in the

635 murine lineage prior to the divergence of the mouse and the rat, (b) depending on the genomic

636 context, most insertions were neutral or mildly deleterious due to their CR binding and mild

637 regulatory activity in new genomic locations, and (c) a small subset of elements were tolerated or

638 advantageous due to their proximity to existing circadian regulatory elements and have been co-

639 opted as circadian enhancers either through binding site turnover, introduction of regulatory

640 redundancy and buffering, or rarely by wiring a new gene into the circadian regulatory network

641 (Fig. 6B).

642

643 Our study of gradual emergence of cis-regulatory activity within RSINE1 suggests an

644 alternative to the copy-and-paste model of TE co-option whereby a TE inserts a ready-made

645 enhancer module in a location where that activity becomes immediately beneficial [51,105-107]. 
646 While this is an attractive model, such cases are likely to be rare because TEs carrying such

647 ready-made cis-regulatory modules, such as those found within the long terminal repeats of

648 retrotransposons and endogenous retroviruses, are substantially more likely to have deleterious

649 effects upon insertion than they are to be adaptive. Instead, TEs with little to no intrinsic cis-

650 regulatory activity upon insertion are more likely to be tolerated near genes and existing cis-

651 regulatory elements. The 'low regulatory profile' of these TEs might enable them to achieve

652 higher copy number and disperse, on a large scale, proto-motifs poised for the emergence of new

653 cis-regulatory elements. SINEs make excellent candidates for this model because they are short,

654 noncoding, and typically derived from tRNA and other Pol III transcripts, thereby reducing their

655 capacity to interfere with Pol II-regulation. Perhaps thanks to this property, SINEs have attained

656 enormous copy numbers in virtually all mammalian genomes and they accumulate closer to

657 genes than other types of TEs such as LINEs or ERVs $[39,40,50,108]$. It may not be coincidental

658 that many studies have identified ancient SINEs co-opted as enhancers and other complex cis-

659 regulatory elements [109-116], but it has been difficult to discern features of these elements that

660 promoted their exaptation. We speculate that we have caught RSINE1 at an early stage of the co-

661 option process and that the principles uncovered herein illuminate how seemingly benign TEs

662 like SINEs have been a pervasive source of cis-regulatory elements in vertebrate evolution. 


\section{Methods}

\section{Reanalysis of sequencing data.}

665 Fastq files were downloaded from SRA (Table 1). Reads were trimmed and filtered with 666 cutadapt [117] (arguments: -a AGATCGGAAGAGCACACGTCTGAACTCCAGTCAC; -A

667 AGATCGGAAGAGCGTCGTGTAGGGAAAGAGTGTAGATCTCGGTGGTCGCCGTATCA

668 TT; --minimum-length=25), and aligned to the mm10 reference genome using bowtie2 [118] in

669 paired end mode where applicable (arguments: --local; --very-sensitive-local; --no-unal; --no-

670 mixed; --no-discordant; -I 10; -X 1000). SOLiD reads were aligned using bowtie [119] in

671 colorspace (arguments: -C; -m 1). Alignment files were processed, sorted, and filtered for

672 uniquely mapping reads $(\mathrm{q}>1)$ using samtools [120] and PCR duplicate reads removed with

673 Picard MarkDuplicates. Peaks were called using macs2 [121] (arguments: -g mm; --call-

674 summits).

675

\section{Genomic interval analyses.}

677 All intersections of sets of genomic intervals were performed using bedtools intersect [122]

678 (arguments: -wo; -f 0.5 ), which requires that $50 \%$ of a feature in the query file overlaps a feature

679 in the subject file in order to record an intersection. Non-intersecting regions were separately

680 determined (arguments: -v; -f 0.5). Sets of CR ChIP-seq peak coordinates were intersected as

681 queries against the output of RepeatMasker [75], which was retrieved from the UCSC genome

682 browser and filtered to remove low complexity regions and simple repeats. All calculations of

683 distances between genomic intervals was calculated using bedtools closest (arguments: $-\mathrm{d}$ ). 


\section{Expression profiles and heatmaps.}

686 Normalized signal coverage files were generated using DeepTools bamCoverage [123]

687 (arguments: --binSize 10; --normalizeTo1x 2150570000). Single end reads were extended to

688 match the average library insert fragment size reported at publication; empirically determined

689 fragment size was used when paired-end sequence data was available. The scaling normalization

690 employed here presents coverage in each bin as a ratio of observed coverage to expected genome

691 wide coverage assuming equal distribution of reads (1x coverage), and accounts for the

692 mappable size of the genome, read depth, and read length. Profile meta-plots and heatmaps were

693 generated using DeepTools [123]. Coverage matrixes were generated using computeMatrix in

694 reference-point mode (arguments: --referencePoint center; --missingDataAsZero). Heatmaps

695 were created with plotHeatmap and profiles were created with plotProfile (arguments: --plotType

696 se). Quantification of signal in peaks was performed by using bigWigSummary to extract mean

697 coverage at each genomic interval; followed by statistical testing in R using the packages

698 kruskal.test and dunn.test. For visualization purposes, when plotting heatmaps designed to

699 compare between sets of intervals with different sample sizes (for example, RABS-RSINE1

$700 \mathrm{n}=328$ and Non-RABS-RSINE1 $\mathrm{n}=115,806)$, the larger set of intervals was subsampled to the

701 match the smallest set of intervals prior to matrix computation.

702

703 Gene Ontology Analysis

704 Enrichment of mouse phenotypes with each group of genomic intervals was calculated by

705 GREAT [77] using the two nearest genes within $1 \mathrm{Mb}$ of each E-Box motif. 
707 Repeat element enrichment.

708 Enrichment was calculated as described [78] (https://github.com/4ureliek/TEanalysis) by

709 shuffling TEs such that the distance to the nearest TSS and number of elements on each

710 chromosome was maintained. These shuffled TEs were intersected with the mm10

711 RepeatMasker annotations [75] to determine the number of expected intersections from each TE

712 and TE family. This procedure was bootstrapped 1000 times. A binomial test was then

713 performed to determine significance, comparing the average expectation to the observed value.

715 Consensus Alignment

716 Consensus sequences of RSINE1, B4, and B4A were obtained from Repbase [74] and aligned

717 using Clustal Omega [124]. The resulting alignment was visualized and manually curated using

718 Jalview [125]. Relevant motifs were identified manually.

$720 \quad$ Motif analysis.

721 Sequences of bound, OC unbound, and all RSINE1 elements were retrieved using bedtools

722 getfasta [122]. Unbiased motif discovery was performed using DREME [126] with bound

723 RSINE1s as primary sequences, and both OC unbound and all RSINE1s as control sequences,

724 sequentially. This procedure was repeated using the $500 \mathrm{bp}$ flanks (left and right) of each

725 RSINE1 element in each set. Locations of E-box (CACRTG), RORE (RGGTCA), and D-Box

726 (TTATGYAA) motifs were predicted genome wide using FIMO [127] (arguments: --thresh 0.01;

727 --max-stored-scores 10000000). Bedtools merge [122] was used to merge overlapping motifs and

728 average the FIMO score over the merged region, and the utility bedGraphToBigWig was used to 
729 generate coverage tracks where the coverage represents genome wide motif occurrence weighted

730 by score. Heatmaps were generated as above. The distance to the nearest E-Box motif from each

731 set of intervals (peaks and repeats) was calculated using bedtools closest [122] (arguments: -d),

732 and E-Boxes which fall within the boundaries of the peak (and therefore are distance $=0$ ) were

733 retained. For analysis of distance between motifs, we filtered coordinates of predicted motifs by

734 FIMO log-odds score, retaining only motifs with the highest possible score. Bedtools intersect

735 (arguments: -f 1; -wa) reported motifs that completely overlap with a given set of repeats; the

736 distance from these overlapping motifs to the nearest non-overlapping motif was calculated using

737 bedtools closest (arguments: -io; -d).

\section{Conservation analysis.}

740 We attempted to map sets of genomic regions from the mm10 genome to the hg38 or $\mathrm{rn} 6$

741 genomes using liftOver (Kuhn et al., 2013) using appropriate chains, and counted the fraction of

742 intervals which did or did not lift over from each set of elements.

\section{RSINE1 phylogeny.}

745 We began with sequences of the 328 bound RSINE1 elements, as well as 1000 randomly

746 selected elements from the set all RSINE1s in the mm10 genome assembly $(\mathrm{n}=115,806)$. We

747 aligned these sequences using Clustal Omega [124] with default settings. From this alignment,

748 we calculated a maximum likelihood tree with FastTree 2 [128] using default settings for

749 nucleotide alignments. The tree was visualized with ggtree [129] and branches were colored by

750 their designation as bound or unbound RSINE1 elements. 


\section{Mouse/Rat Motif Comparison}

753 We started with the set of CR bound RSINE1 elements $(\mathrm{n}=328)$ and unbound elements residing

754 in open chromatin $(n=4583)$ and attempted to assign syntenic orthologs of each element using

755 liftOver [84]. We were able to find orthologous regions for 195 bound RSINE1 elements

$756(59.5 \%)$ and 2662 unbound elements (58.1\%). We queried the sequences of each of these

757 elements using bedtools getfasta [122] from the mm10 and rn6 genome assemblies, and used

758 DREME [126] as above to find motifs enriched in orthologous bound RSINE1 elements when

759 compared to unbound RSINE1 elements.

760

761 Tissue Specific BMAL1 Analysis

762 We started with coordinates of BMAL1 binding sites in the liver, kidney, and heart [72] in the

$763 \mathrm{~mm} 10$ genome assembly. We performed an intersection as described above (requiring $50 \%$

764 overlap of a given peak with a repetitive element to designate that peak as a RABS) between

765 each of these sets of coordinates and the output of RepeatMasker [75] which was retrieved from

766 the UCSC genome browser and filtered to remove low complexity regions and simple repeats.

767 We counted the number of Non-RABS, RABS, and RSINE1-RABS and plotted the fraction each

768 category represents with respect to the total set of peaks, and the fraction of RABS which are

769 RSINE1-derived.

770

\section{$771 \quad$ Luciferase Reporter Assays}

772 We accessed the RSINE1 consensus sequence from Repbase [74] and created an "in silico

773 evolved" version by changing a total of $7 \mathrm{nt}$ to "perfect" the E-Box and RORE motifs

774 highlighted in Fig. 4A (RORE 1: unchanged; RORE 2: AGTTCG>AGGTCA; RORE 3: 
775 AGGAGA>AGGTCA; E-Box 1: CACATG>CACGTG; E-Box 2\&3: CACGCG>CACGTG).

776 We also accessed, from the mm10 genome assembly, the sequences of Tmem267 and Aaed1

777 RSINE1 elements highlighted in Fig. S6, both with and without $100 \mathrm{nt}$ of flanking sequence on

778 the 5' and $3^{\prime}$ ends. All of these sequences were synthesized as gBlocks ${ }^{\circledR}$ Gene Fragments

779 (Integrated DNA Technologies, Coralville, IA, USA) and cloned into pGL3::PRO (Promega

780 Corporation, Madison, WI, USA) downstream of the luciferase gene. To generate vectors of

781 Tmem267 and Aaed1 RSINE1 flanking sequence only, existing vectors were amplified by PCR

782 using Q5 High-Fidelity DNA Polymerase (New England Biolabs, Ipswich, MA, USA) with

783 primers designed to delete the RSINE1 element by site directed mutagenesis. All vectors were

784 validated by Sanger sequencing. Hepa 1-6 cells were obtained from ATCC and were subject to

785 co-transfection with $500 \mathrm{ng}$ of each of the constructs described above in conjunction with $500 \mathrm{ng}$

786 pRL::SV40 Renilla Luciferase Control Vector (Promega Corporation, Madison, WI, USA) using

787 Lipofectamine-2000 (Thermo Fisher Scientific, Waltham, MA, USA). After 18h, firefly and

788 Renilla luciferase luminescence was measured using the Dual-Glo® Luciferase Assay System

789 (Promega Corporation, Madison, WI, USA). Firefly luminescence values were normalized to

790 Renilla luminescence and compared to the empty pGL3::PRO vector. 


\section{Declarations}

794 Ethics approval and consent to participate

795 Not Applicable.

\section{Consent for publication}

798 Not Applicable.

801 All data used in this study was previously published and accession numbers can be found in 802 Table 1.

\section{Competing interests}

805 The authors declare that they have no competing interests.

806

\section{Funding}

808 This work was supported by awards R35-GM122550 and U01-HG009391 from the National

809 Institutes of Health to CF JJ was supported by NHGRI fellowship F31HG010820. HS was

810 supported by the University of Utah Undergraduate Research Opportunities Program. The

811 content is solely the responsibility of the authors and does not necessarily represent the official

812 views of the National Institutes of Health.

\section{Authors' contributions}

$815 \mathrm{CF}, \mathrm{JJ}$, and HS conceptualized the study. JJ and HS performed genomic data analysis. JJ

816 conducted reporter assays. JJ, CF, and HS wrote the manuscript. All authors read and approved

817 the final manuscript.

818

\section{Acknowledgements}

820 The authors acknowledge Tianyi Zhang and Fanliu Kong for technical assistance with cloning, 821 and thank members of the Feschotte Lab and Lis Lab for helpful discussions. 


\section{References}

824 1. Carroll SB, Prud'homme B, Gompel N. Regulating evolution. Sci Am. 2008;298:60-7.

825 2. King MC, Wilson AC. Evolution at two levels in humans and chimpanzees. Science (80- ).

$826 \quad 1975 ; 188: 107-16$.

827 3. Wagner GP, Lynch VJ. Evolutionary novelties. Curr Biol. 2010;20:48-52.

828 4. Romero IG, Ruvinsky I, Gilad Y. Comparative studies of gene expression and the evolution of 829 gene regulation. Nat Rev Genet. 2012;13:505-16.

830 5. Reilly SK, Noonan JP. Evolution of Gene Regulation in Humans. Annu Rev Genomics Hum Genet. 2016;17:45-67.

832 6. Stern DL, Orgogozo V. The loci of evolution: How predictable is genetic evolution? Evolution (N Y). 2008;62:2155-77.

834 7. Wray GA. The evolutionary significance of cis-regulatory mutations. Nat Rev Genet. 2007;8:206-16.

836 8. Rebeiz M, Tsiantis M. Enhancer evolution and the origins of morphological novelty. Curr Opin Genet Dev. 2017;45:115-23.

9. Pennacchio LA, Bickmore W, Dean A, Nobrega MA, Bejerano G. Enhancers: Five essential 839 questions. Nat Rev Genet. 2013;14:288-95.

840 10. Rubinstein M, de Souza FSJ. Evolution of transcriptional enhancers and animal diversity.

$841 \quad$ Philos Trans R Soc B Biol Sci. 2013;368:20130017.

842 11. Villar D, Flicek P, Odom DT. Evolution of transcription factor binding in metazoans843 mechanisms and functional implications. Nat Rev Genet. 2014;15:221-33.

844 12. Wittkopp PJ. Variable transcription factor binding: A mechanism of evolutionary change. 
PLoS Biol. 2010;8:e1000342.

846 13. Levine M, Cattoglio C, Tjian R. Looping back to leap forward: Transcription enters a new $847 \quad$ era. Cell. 2014;157:13-25.

848 14. Schoenfelder S, Fraser P. Long-range enhancer-promoter contacts in gene expression 849 control. Nat Rev Genet. 2019;20:437-55.

850 15. Arnold CD, Gerlach D, Stelzer C, Boryń ŁM, Rath M, Stark A. Genome-wide quantitative 851 enhancer activity maps identified by STARR-seq. Science (80- ). 2013;339:1074-7.

852 16. Heidari N, Phanstiel DH, He C, Grubert F, Jahanbani F, Kasowski M, et al. Genome-wide 853 map of regulatory interactions in the human genome. Genome Res. 2014;24:1905-17.

854 17. Noonan JP. Regulatory DNAs and the evolution of human development. Curr Opin Genet 855 Dev. 2009;19:557-64.

856 18. Blow MJ, McCulley DJ, Li Z, Zhang T, Akiyama JA, Holt A, et al. ChIP-seq identification 857 of weakly conserved heart enhancers. Nat Genet. 2010;42:806-12.

858 19. Cotney J, Leng J, Yin J, Reilly SK, Demare LE, Emera D, et al. XThe evolution of lineage859 specific regulatory activities in the human embryonic limb. Cell. 2013;154:185.

860 20. Kunarso G, Chia NY, Jeyakani J, Hwang C, Lu X, Chan YS, et al. Transposable elements 861 have rewired the core regulatory network of human embryonic stem cells. Nat Genet. $862 \quad 2010 ; 42: 631-4$.

863 21. Schmidt D, Wilson MD, Ballester B, Schwalie PC, Brown GD, Marshall A, et al. Five864 vertebrate ChlP-seq reveals the evolutionary dynamics of transcription factor binding. $865 \quad$ Science (80- ). 2010;328:1036-40.

866 22. Shen Y, Yue F, Mc Cleary DF, Ye Z, Edsall L, Kuan S, et al. A map of the cis-regulatory 867 sequences in the mouse genome. Nature. 2012;488:116-20. 
23. Stefflova K, Thybert D, Wilson MD, Streeter I, Aleksic J, Karagianni P, et al. mammals. Cell. 2013;154:530-40.

871 24. Vierstra J, Rynes E, Sandstrom R, Zhang M, Canfield T, Scott Hansen R, et al. Mouse regulatory DNA landscapes reveal global principles of cis-regulatory evolution. Science

25. Douglas AT, Hill RE. Variation in vertebrate Cis-regulatory elements in evolution and (80- ). 2014;346:1007-12. Development and Evolution. Cell. 2016;167:1170-87. landscape in human embryonic stem cells. Stem Cell Res. 2019;37:101456.

880 28. Villar D, Berthelot C, Aldridge S, Rayner TF, Lukk M, Pignatelli M, et al. Enhancer evolution across 20 mammalian species. Cell. 2015;160:554-66.

29. Berthelot C, Villar D, Horvath JE, Odom DT, Flicek P. Complexity and conservation of regulatory landscapes underlie evolutionary resilience of mammalian gene expression.

885 30. Dickel DE, Ypsilanti AR, Pla R, Zhu Y, Barozzi I, Mannion BJ, et al. Ultraconserved Enhancers Are Required for Normal Development. Cell. 2018;172:491-499.e15.

887 31. Li S, Kvon EZ, Visel A, Pennacchio LA, Ovcharenko I. Stable enhancers are active in 888 development, and fragile enhancers are associated with evolutionary adaptation. Genome

890 32. Eichenlaub MP, Ettwiller L. De novo genesis of enhancers in vertebrates. PLoS Biol. 
2011;9:e1001188.

892 33. Klein JC, Keith A, Agarwal V, Durham T, Shendure J. Functional characterization of

893 enhancer evolution in the primate lineage. Genome Biol. 2018;19:1-13.

894 34. Trizzino M, Park Y, Holsbach-Beltrame M, Aracena K, Mika K, Caliskan M, et al.

895 Transposable elements are the primary source of novelty in primate gene regulation.

$896 \quad$ Genome Res. 2017;27:1623-33.

897 35. Young RS. Lineage-specific genomics: Frequent birth and death in the human genome: The

898 human genome contains many lineage-specific elements created by both sequence and

899 functional turnover. BioEssays. 2016;38:654-63.

900 36. Wells JN, Feschotte C. A Field Guide to Eukaryotic Transposable Elements. Annu Rev

901 Genet. 2020;54:annurev-genet-040620-022145.

902 37. de Koning APJ, Gu W, Castoe TA, Batzer MA, Pollock DD. Repetitive elements may

903 comprise over two-thirds of the human genome. PLoS Genet. 2011;7:e1002384.

904 38. Mikkelsen TS, Wakefield MJ, Aken B, Amemiya CT, Chang JL, Duke S, et al. Genome of

905 the marsupial Monodelphis domestica reveals innovation in non-coding sequences.

$906 \quad$ Nature. 2007;447:167-77.

907 39. Lander ES, Linton LM, Birren B, Nusbaum C, Zody MC, Baldwin J, et al. Initial sequencing 908 and analysis of the human genome. Nature. 2001;409:860-921.

909 40. Waterston RH, Lindblad-Toh K, Birney E, Rogers J, Abril JF, Agarwal P, et al. Initial 910 sequencing and comparative analysis of the mouse genome. Nature. 2002;420:520-62.

911 41. Kapusta A, Suh A, Feschotte C. Dynamics of genome size evolution in birds and mammals.

$912 \quad$ Proc Natl Acad Sci. 2017;114:E1460-9.

913 42. Sudmant PH, Rausch T, Gardner EJ, Handsaker RE, Abyzov A, Huddleston J, et al. An 

integrated map of structural variation in 2,504 human genomes. Nature. 2015;526:75-81.

915 43. Feschotte C. Transposable elements and the evolution of regulatory networks. Nat Rev $916 \quad$ Genet. 2008;9:397-405.

917 44. Chuong EB, Elde NC, Feschotte C. Regulatory activities of transposable elements: From 918 conflicts to benefits. Nat Rev Genet. 2017;18:71-86.

919 45. Sundaram V, Choudhary MNK, Pehrsson E, Xing X, Fiore C, Pandey M, et al. Functional 920 cis-regulatory modules encoded by mouse-specific endogenous retrovirus. Nat Commun. $921 \quad 2017 ; 8: 1-12$.

46. Sundaram V, Wysocka J. Transposable elements as a potent source of diverse cis-regulatory sequences in mammalian genomes. Philos Trans R Soc Lond B Biol Sci. 2020;375:20190347.

925 47. Rebollo R, Romanish MT, Mager DL. Transposable elements: An abundant and natural 926 source of regulatory sequences for host genes. Annu Rev Genet. 2012;46:21-42.

927 48. Sundaram V, Cheng Y, Ma Z, Li D, Xing X, Edge P, et al. Widespread contribution of 928 transposable elements to the innovation of gene regulatory networks. Genome Res. $929 \quad 2014 ; 24: 1963-76$.

930 49. Wang T, Zeng J, Lowe CB, Sellers RG, Salama SR, Yang M, et al. Species-specific 931 endogenous retroviruses shape the transcriptional network of the human tumor suppressor protein p53. Proc Natl Acad Sci. 2007;104:18613-8.

933 50. Schmidt D, Schwalie PC, Wilson MD, Ballester B, Gonalves Â, Kutter C, et al. Waves of 934 retrotransposon expansion remodel genome organization and CTCF binding in multiple 935 mammalian lineages. Cell. 2012;148:335-48.

936 51. Chuong EB, Elde NC, Feschotte C. Regulatory evolution of innate immunity through co- 
option of endogenous retroviruses. Science (80- ). 2016;351:1083-7.

938 52. Bourque G, Leong B, Vega VB, Chen X, Yen LL, Srinivasan KG, et al. Evolution of the 939 mammalian transcription factor binding repertoire via transposable elements. Genome $940 \quad$ Res. 2008;18:1752-62.

941 53. Jacques P-É, Jeyakani J, Bourque G. The majority of primate-specific regulatory sequences $942 \quad$ are derived from transposable elements. PLoS Genet. 2013;9:e1003504.

943 54. Gibbs RA, Weinstock GM, Metzker ML, Muzny DM, Sodergren EJ, Scherer S, et al.

944 Genome sequence of the Brown Norway rat yields insights into mammalian evolution.

$945 \quad$ Nature. 2004;428:493-520.

946 55. Lynch VJ, Leclerc RD, May G, Wagner GP. Transposon-mediated rewiring of gene

947 regulatory networks contributed to the evolution of pregnancy in mammals. Nat Genet.

$948 \quad 2011 ; 43: 1154-9$.

949 56. Mohawk JA, Green CB, Takahashi JS. Central and peripheral circadian clocks in mammals.

$950 \quad$ Annu Rev Neurosci. 2012;35:445-62.

951 57. Takahashi JS. Transcriptional architecture of the mammalian circadian clock. Nat Rev Genet. $952 \quad 2017 ; 18: 164-79$.

953 58. Welsh DK, Takahashi JS, Kay SA. Suprachiasmatic nucleus: Cell autonomy and network $954 \quad$ properties. Annu Rev Physiol. 2009;72:551-77.

955 59. Rey G, Cesbron F, Rougemont J, Reinke H, Brunner M, Naef F. Genome-wide and phase956 specific DNA-binding rhythms of BMAL1 control circadian output functions in mouse 957 liver. PLoS Biol. 2011;9:e1000595.

958 60. Gekakis N, Staknis D, Nguyen HB, Davis FC, Wilsbacner LD, King DP, et al. Role of the 959 CLOCK protein in the mammalian circadian mechanism. Science (80- ). 1998;280:1564- 
9.

961 61. Kume K, Zylka MJ, Sriram S, Shearman LP, Weaver DR, Jin X, et al. mCRY1 and mCRY2 are essential components of the negative limb of the circadian clock feedback loop. Cell.

62. Menet JS, Pescatore S, Rosbash M. CLOCK: BMAL1 is a pioneer-like transcription factor. Genes Dev. 2014;28:8-13. CLOCK:BMAL1. PLOS Genet. 2018;14:e1007156.

64. Le Martelot G, Claudel T, Gatfield D, Schaad O, Kornmann B, Lo Sasso G, et al. REVERB $\alpha$ participates in circadian SREBP signaling and bile acid homeostasis. PLoS Biol.

65. Turek FW, Joshu C, Kohsaka A, Lin E, Ivanova G, McDearmon E, et al. Obesity and metabolic syndrome in circadian Clock mutant nice. Science (80- ). 2005;308:1043-5.

973 66. Zhao X, Cho H, Yu RT, Atkins AR, Downes M, Evans RM. Nuclear receptors rock around the clock. EMBO Rep. 2014;15:518-28.

975 67. Koike N, Yoo SH, Huang HC, Kumar V, Lee C, Kim TK, et al. Transcriptional architecture and chromatin landscape of the core circadian clock in mammals. Science (80- ). 2012;338:349-54. regulatory logic of the diurnal cycle in the mouse liver. PLOS Biol. 2017; 15:e2001069.

69. Fang B, Everett LJ, Jager J, Briggs E, Armour SM, Feng D, et al. Circadian enhancers coordinate multiple phases of rhythmic gene transcription in vivo. Cell. 2014;159:1140- 
983 70. Boergesen M, Pedersen TA, Gross B, van Heeringen SJ, Hagenbeek D, Bindesbøll C, et al.

984 Genome-wide profiling of liver $\mathrm{X}$ receptor, retinoid X receptor, and peroxisome

985 proliferator-activated receptor $\alpha$ in mouse liver reveals extensive sharing of binding sites.

$986 \quad$ Mol Cell Biol. 2012;32:852-67.

987 71. Cho H, Zhao X, Hatori M, Yu RT, Barish GD, Lam MT, et al. Regulation of circadian

988 behaviour and metabolism by REV-ERB- $\alpha$ and REV-ERB- $\beta$. Nature. 2012;485:123-7.

989 72. Beytebiere JR, Trott AJ, Greenwell BJ, Osborne CA, Vitet H, Spence J, et al. Tissue-specific

990 BMAL1 cistromes reveal that rhythmic transcription is associated with rhythmic

991 enhancer-enhancer interactions. Genes Dev. 2019;33:294-309.

992 73. Huang L, Damle SS, Booten S, Singh P, Sabripour M, Hsu J, et al. Partial Hepatectomy

993 Induced Long Noncoding RNA Inhibits Hepatocyte Proliferation during Liver

$994 \quad$ Regeneration. PLoS One. 2015;10:e0132798.

995 74. Jurka J, Kapitonov VV, Pavlicek A, Klonowski P, Kohany O, Walichiewicz J. Repbase

996 Update, a database of eukaryotic repetitive elements. Cytogenet Genome Res.

$997 \quad 2005 ; 110: 462-7$.

998 75. Smit A, Hubley R, Green P. RepeatMasker Open-4.0 [Internet]. 2013. Available from:

999 http://www.repeatmasker.org

1000 76. Kent WJ, Sugnet CW, Furey TS, Roskin KM, Pringle TH, Zahler AM, et al. The Human

1001 Genome Browser at UCSC. Genome Res. 2002;12:996-1006.

1002 77. McLean CY, Bristor D, Hiller M, Clarke SL, Schaar BT, Lowe CB, et al. GREAT improves

1003 functional interpretation of cis-regulatory regions. Nat Biotechnol. 2010;28:495-501.

1004 78. Kapusta A, Kronenberg Z, Lynch VJ, Zhuo X, Ramsay LA, Bourque G, et al. Transposable

1005 Elements Are Major Contributors to the Origin, Diversification, and Regulation of 
Vertebrate Long Noncoding RNAs. PLoS Genet. 2013;9.

1007

1008

1009

1010

1011

1012

1013

1014

1015

1016

1017

1018

1019

1020

1021

1022

1023

1024

1025

1026

1027

1028

79. Hubley R, Finn RD, Clements J, Eddy SR, Jones TA, Bao W, et al. The Dfam database of repetitive DNA families. Nucleic Acids Res. 2016;44:D81-9.

80. Lee IY, Westaway D, Smit AFA, Wang K, Seto J, Chen L, et al. Complete genomic sequence and analysis of the prion protein gene region from three mammalian species. Genome Res. 1998;8:1022-37.

81. Giguere V, Tini M, Flock G, Ong E, Evans RM, Otulakowski G. Isoform-specific aminoterminal domains dictate DNA-binding properties of $\operatorname{ROR} \alpha$, a novel family of orphan hormone nuclear receptors. Genes Dev. 1994;8:538-53.

82. Guillaumond F, Dardente H, Giguère V, Cermakian N. Differential control of Bmal1 circadian transcription by REV-ERB and ROR nuclear receptors. J Biol Rhythms. 2005;20:391-403.

83. Shen Q, Bai Y, Chang KCN, Wang Y, Burris TP, Freedman LP, et al. Liver X receptorretinoid X receptor (LXR-RXR) heterodimer cistrome reveals coordination of LXR and AP1 signaling in keratinocytes. J Biol Chem. 2011;286:14554-63.

84. Kuhn RM, Haussler D, James Kent W. The UCSC genome browser and associated tools. Brief Bioinform. 2013;14:144-61.

85. Mitsui S, Yamaguchi S, Matsuo T, Ishida Y, Okamura H. Antagonistic role of E4BP4 and PAR proteins in the circadian oscillatory mechanism. Genes Dev. 2001;15:995-1006.

86. Todd CD, Deniz Ö, Taylor D, Branco MR. Functional evaluation of transposable elements as enhancers in mouse embryonic and trophoblast stem cells. Elife. 2019;8:e44344.

87. Duhl DMJ, Vrieling H, Miller KA, Wolff GL, Barsh GS. Neomorphic agouti mutations in obese yellow mice. Nat Genet. 1994;8:59-65. 
1029 88. Morgan HD, Sutherland HGE, Martin DIK, Whitelaw E. Epigenetic inheritance at the agouti $1030 \quad$ locus in the mouse. Nat Genet. 1999;23:314-8.

1031 89. Whitelaw E, Martin DIK. Retrotransposons as epigenetic mediators of phenotypic variation 1032 in mammals. Nat Genet. 2001;27:361-5.

1033 90. Duez H, Staels B. The nuclear receptors Rev-erbs and RORs integrate circadian rhythms and 1034 metabolism. Diabetes Vasc Dis Res. 2008;5:82-8.

1035 91. Solt LA, Kojetin DJ, Burris TP. The REV-ERBs and RORs: Molecular links between 1036 circadian rhythms and lipid homeostasis. Future Med Chem. 2011;3:623-38.

1037 92. Grow EJ, Flynn RA, Chavez SL, Bayless NL, Wossidlo M, Wesche DJ, et al. Intrinsic retroviral reactivation in human preimplantation embryos and pluripotent cells. Nature. retrotransposon binds the transcription factors dioxin receptor and Slug and regulates

94. Simmen MW. Genome-scale relationships between cytosine methylation and dinucleotide abundances in animals. Genomics. 2008;92:33-40.

95. Bird AP. DNA methylation and the frequency of CpG in animal DNA. Nucleic Acids Res.

$1046 \quad$ 1980;8:1499-504.

1047 96. Antequera F, Bird A. Number of CpG islands and genes in human and mouse. Proc Natl $1048 \quad$ Acad Sci. 1993;90:11995-9.

1049 97. Ichiyanagi K, Li Y, Watanabe T, Ichiyanagi T, Fukuda K, Kitayama J, et al. Locus- and 1050 domain-dependent control of DNA methylation at mouse B1 retrotransposons during 1051 male germ cell development. Genome Res. 2011;21:2058-66. 
1052

1053

1054

1055

1056

1057

1058

1059

1060

1061

1062

1063

1064

1065

1066

1067

1068

1069

1070

1071

1072

1073

1074

98. Molaro A, Falciatori I, Hodges E, Aravin AA, Marran K, Rafii S, et al. Two waves of de novo methylation during mouse germ cell development. Genes Dev. 2014;28:1544-9.

99. Popp C, Dean W, Feng S, Cokus SJ, Andrews S, Pellegrini M, et al. Genome-wide erasure of DNA methylation in mouse primordial germ cells is affected by AID deficiency. Nature. 2010;463:1101-5.

100. Zemojtel T, Kielbasa SM, Arndt PF, Behrens S, Bourque G, Vingron M. CpG deamination creates transcription factor-binding sites with high efficiency. Genome Biol Evol. 2011;3:1304-11.

101. Zemojtel T, Kielbasa SM, Arndt PF, Chung HR, Vingron M. Methylation and deamination of CpGs generate p53-binding sites on a genomic scale. Trends Genet. 2009;25:63-6.

102. Emera D, Wagner GP. Transformation of a transposon into a derived prolactin promoter with function during human pregnancy. Proc Natl Acad Sci. 2012;109:11246-51.

103. Cannavò E, Khoueiry P, Garfield DA, Geeleher P, Zichner T, Gustafson EH, et al. Shadow Enhancers Are Pervasive Features of Developmental Regulatory Networks. Curr Biol. 2016;26:38-51.

104. Choudhary MNK, Friedman RZ, Wang JT, Jang HS, Zhuo X, Wang T. Co-opted transposons help perpetuate conserved higher-order chromosomal structures. Genome Biol. 2020;21:16.

105. Dunn-Fletcher CE, Muglia LM, Pavlicev M, Wolf G, Sun M-A, Hu Y-C, et al. Anthropoid primate-specific retroviral element THE1B controls expression of $\mathrm{CRH}$ in placenta and alters gestation length. PLOS Biol. 2018;16:e2006337.

106. Chuong EB, Rumi MAK, Soares MJ, Baker JC. Endogenous retroviruses function as species-specific enhancer elements in the placenta. Nat Genet. 2013;45:325-9. 
1075 107. Pi W, Zhu X, Wu M, Wang Y, Fulzele S, Eroglu A, et al. Long-range function of an

1076 intergenic retrotransposon. Proc Natl Acad Sci. 2010;107:12992-7.

1077 108. Zhang Y, Romanish MT, Mager DL. Distributions of Transposable Elements Reveal

1078 Hazardous Zones in Mammalian Introns. PLoS Comput Biol. 2011;7:e1002046.

1079 109. Notwell JH, Chung T, Heavner W, Bejerano G. A family of transposable elements co-opted 1080 into developmental enhancers in the mouse neocortex. Nat Commun. 2015;6:6644.

1081 110. Lowe CB, Bejerano G, Haussler D. Thousands of human mobile element fragments undergo strong purifying selection near developmental genes. Proc Natl Acad Sci. 2007;104:8005-10.

1084 111. Bejerano G, Lowe CB, Ahituv N, King B, Siepel A, Salama SR, et al. A distal enhancer and an ultraconserved exon are derived from a novel retroposon. Nature. 2006;441:87-90. SINE-Derived Element Constitutes a Unique Modular Enhancer for Mammalian Diencephalic Fgf8. PLoS One. 2012;7:e43785.

113. Nishihara H, Kobayashi N, Kimura-Yoshida C, Yan K, Bormuth O, Ding Q, et al. Coordinately Co-opted Multiple Transposable Elements Constitute an Enhancer for wnt5a Expression in the Mammalian Secondary Palate. PLOS Genet. 2016;12:e1006380.

1092 114. Nishihara H, Smit AFA, Okada N. Functional noncoding sequences derived from SINEs in 1093 the mammalian genome. Genome Res. 2006;16:864-74.

1094 115. Samstein RM, Josefowicz SZ, Arvey A, Treuting PM, Rudensky AY. Extrathymic 1095 generation of regulatory $\mathrm{T}$ cells in placental mammals mitigates maternal-fetal conflict. Cell. 2012;150:29-38.

1097 116. Lam DD, de Souza FSJ, Nasif S, Yamashita M, López-Leal R, Otero-Corchon V, et al. 
Partially Redundant Enhancers Cooperatively Maintain Mammalian Pomc Expression

117. Martin M. Cutadapt removes adapter sequences from high-throughput sequencing reads. EMBnet.journal. 2011;17:10.

118. Langmead B, Salzberg SL. Fast gapped-read alignment with Bowtie 2. Nat Methods. 2012;9:357-9. short DNA sequences to the human genome. Genome Biol. 2009;10:R25. Alignment/Map format and SAMtools. Bioinformatics. 2009;25:2078-9. Protoc. 2012;7:1728-40.

1110 122. Quinlan AR, Hall IM. BEDTools: A flexible suite of utilities for comparing genomic 1111 features. Bioinformatics. 2010;26:841-2.

1112 123. Ramírez F, Dündar F, Diehl S, Grüning BA, Manke T. deepTools: a flexible platform for 1113 exploring deep-sequencing data. Nucleic Acids Res. 2014;42:W187-91.

1114 124. Sievers F, Wilm A, Dineen D, Gibson TJ, Karplus K, Li W, et al. Fast, scalable generation 1115 of high-quality protein multiple sequence alignments using Clustal Omega. Mol Syst $1116 \quad$ Biol. 2011;7:539.

1117 125. Waterhouse AM, Procter JB, Martin DMA, Clamp M, Barton GJ. Jalview Version 2-A multiple sequence alignment editor and analysis workbench. Bioinformatics. 2009;25:1189-91.

1120 126. Bailey TL. DREME: Motif discovery in transcription factor ChIP-seq data. Bioinformatics. 
2011;27:1653-9.

1122 127. Grant CE, Bailey TL, Noble WS. FIMO: Scanning for occurrences of a given motif.

$1123 \quad$ Bioinformatics. 2011;27:1017-8.

1124 128. Price MN, Dehal PS, Arkin AP. FastTree 2 - Approximately maximum-likelihood trees for

1125 large alignments. PLoS One. 2010;5:e9490.

1126 129. Yu G, Lam TT-Y, Zhu H, Guan Y. Two Methods for Mapping and Visualizing Associated 


\section{Supplemental Figures}

A

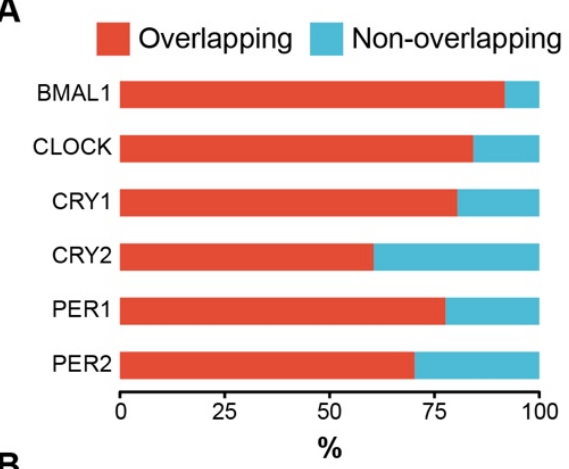

D

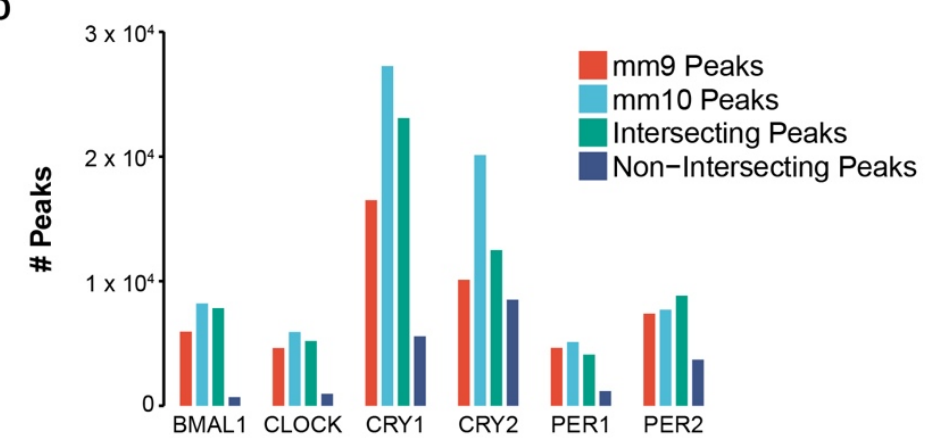

B BMAL1 CLOCK

$\begin{array}{llll}\text { CRY1 } & \text { CRY2 } & \text { PER1 } & \text { PER2 }\end{array}$
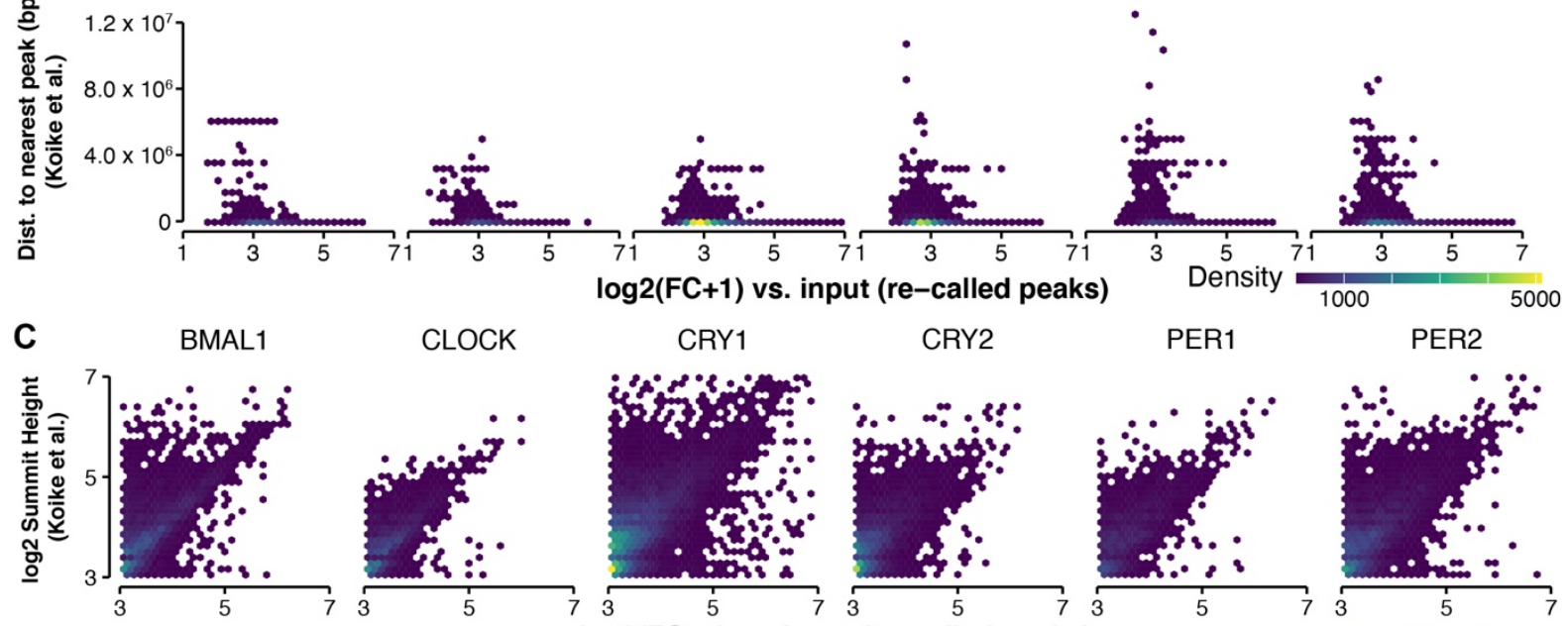

1131 CRY1

CRY2

PER1

PER2

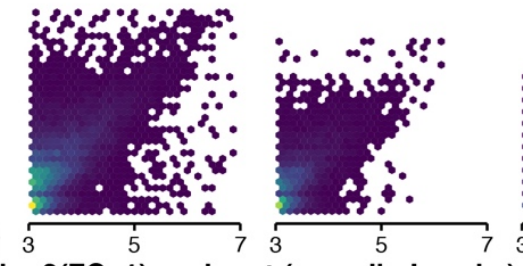

$\log 2(\mathrm{FC}+1)$ vs. input (re-called peaks)

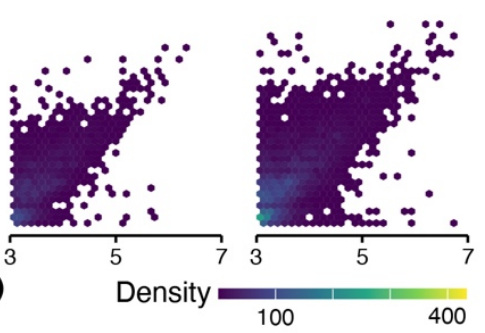

1132 Figure S1: New analysis of Circadian Regulator ChIP-seq data is similar to published

1133 results.

1134 (A) Percentage of peaks determined in the new analysis described in this report that overlap with 1135 peaks in the published set.

1136 (B) Fold-change as reported by macs2 in the re-analysis compared to summit height for each 1137 peak as reported in the published set of peaks.

1138 (C)Fold-change as above for each peak in the new analysis compared to the distance to the 1139 nearest peak in the published set (peaks in the published set were mapped to mm10 $1140 \quad$ coordinates using LiftOver). 
1141 (D) Number of peaks in the published set and in the new analysis presented here for each CR, as 1142 well as the number of peaks which are shared or not shared between the two datasets.

1143 For (C-D), the plot space was divided into hexbins and color-scaled according to the density of

1144 points in each bin to accommodate over-plotting.

1145 

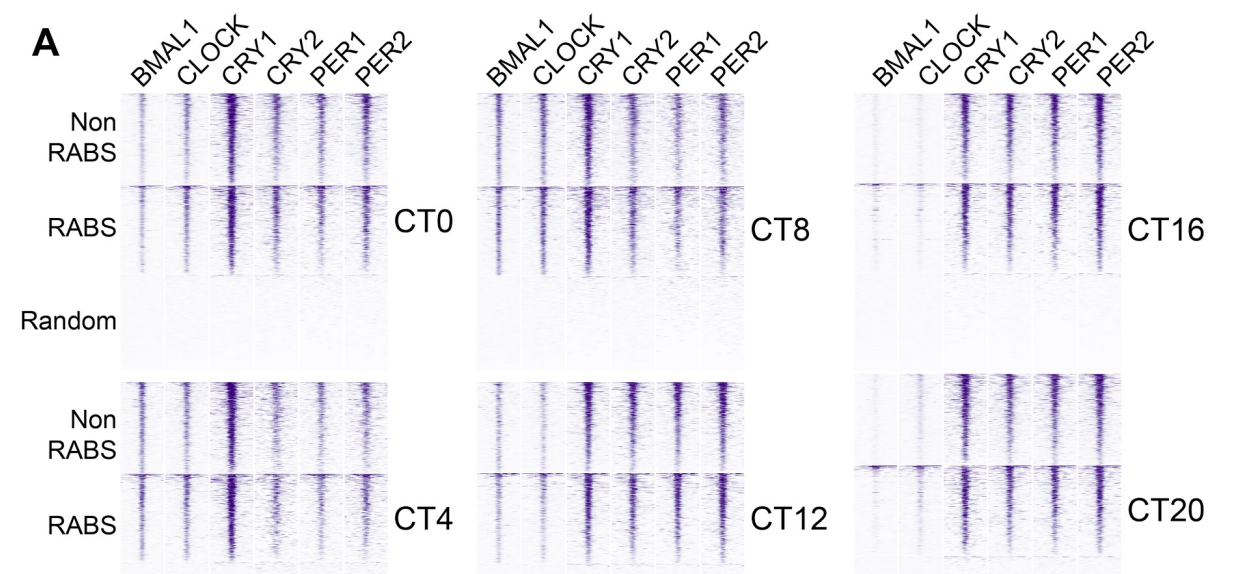

Random

$\longmapsto 1.5 \mathrm{~kb}$

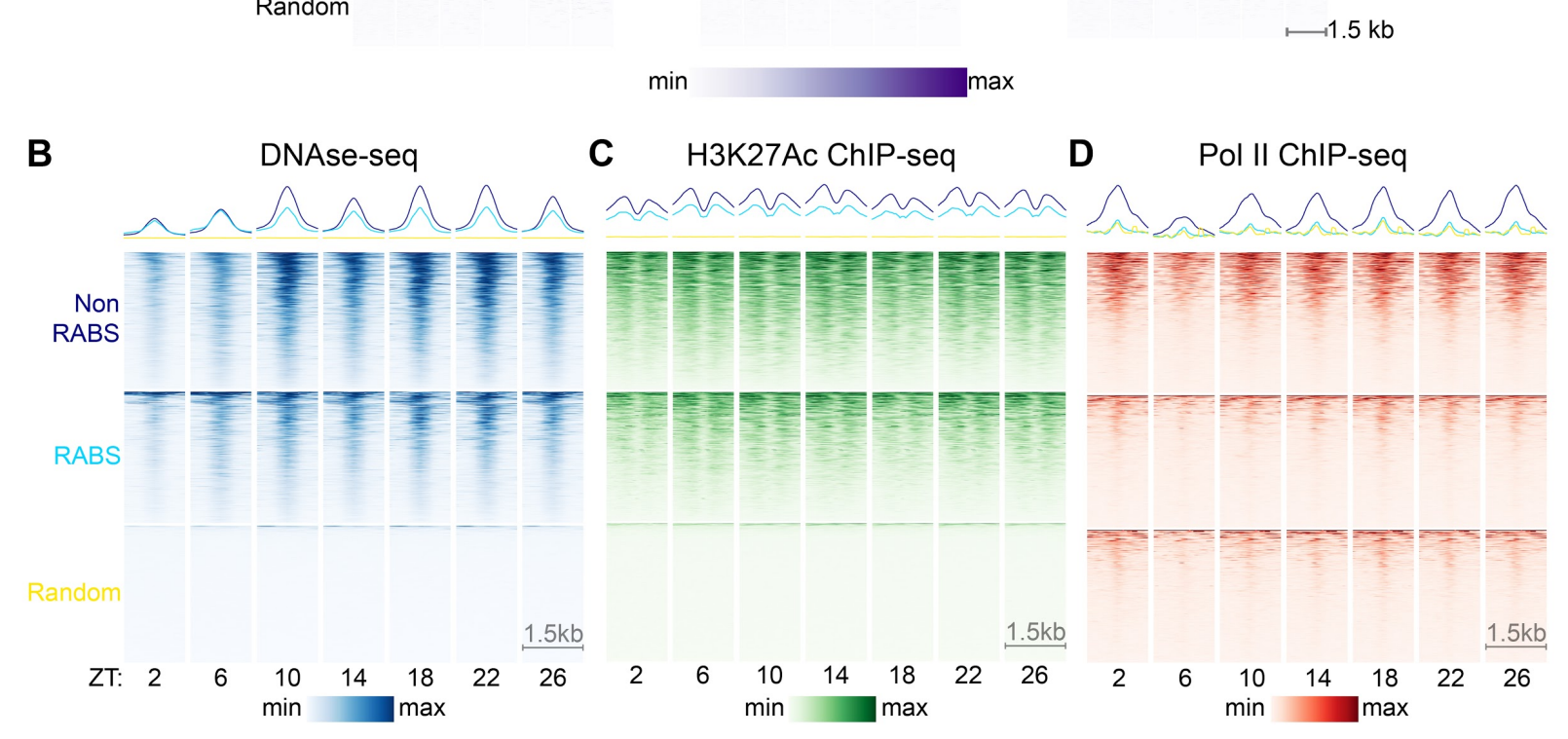

Figure S2: RABS have a similar pattern of CR occupancy but different chromatin

1149 (A) ChIP-seq signal of each CR centered at BMAL1 ChIP-seq peaks at RABS ( $\mathrm{n}=1014)$, randomly selected Non-RABS ( $\mathrm{n}=1014)$, and randomly selected repeats matching the familial composition of RABS ( $\mathrm{n}=1014)$ over Circadian Time (CT).

(B) DNase-seq signal over Zeitgeber time (ZT). Regions as in (A).

(C)H3K27Ac ChIP-seq over ZT. Regions as in (A).

(D) Pol II ChIP-seq at over ZT. Regions as in (A). 
bioRxiv preprint doi: https://doi.org/10.1101/2020.11.09.375469; this version posted November 10,2020 . The copyright holder for this preprint (which was not certified by peer review) is the author/funder, who has granted bioRxiv a license to display the preprint in perpetuity. It is made available under aCC-BY 4.0 International license.

1155 In all panels, heatmaps are sorted by mean signal intensity across all rows in a block of

1156 heatmaps. Color scaling is min-max within each block.

1157 


\section{A E-box}

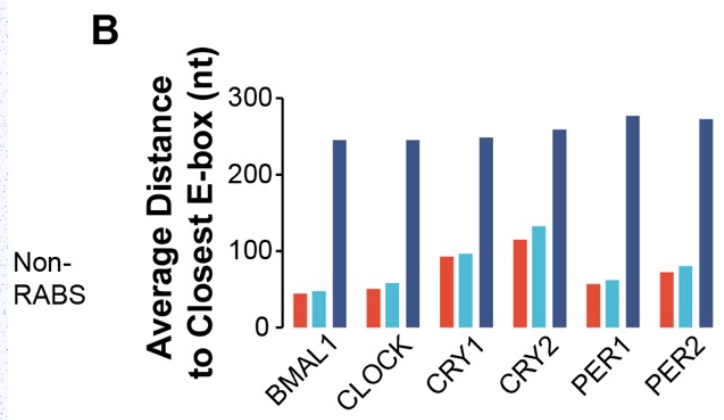

Non-RABS

RABS

Random Repeats

RABS

Random

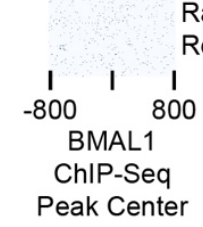

Figure S3: Repeats contribute E-Box motifs.

(A) Occurrence of E-Box motifs at RABS and Non-RABS BMAL1 ChIP-seq peaks, as well as a set of repeats randomly selected to match the familial composition of repeats associated with BMAL1 RABS. Signal strength (color intensity) is relative to motif similarity to the consensus motif.

(B) Average distance to the closest E-Box motif from RABS or Non-RABS ChIP-seq peak centers as well as a randomly selected set of repeats that matches the familial composition of each set of CR RABS. 
A

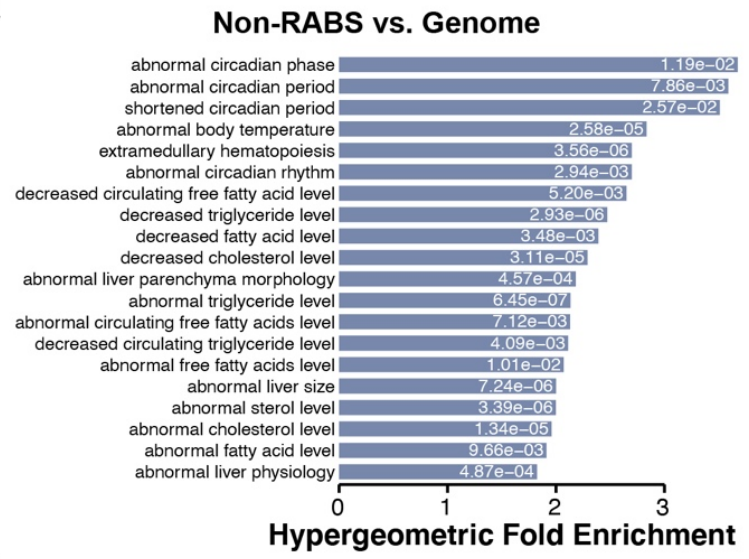

B

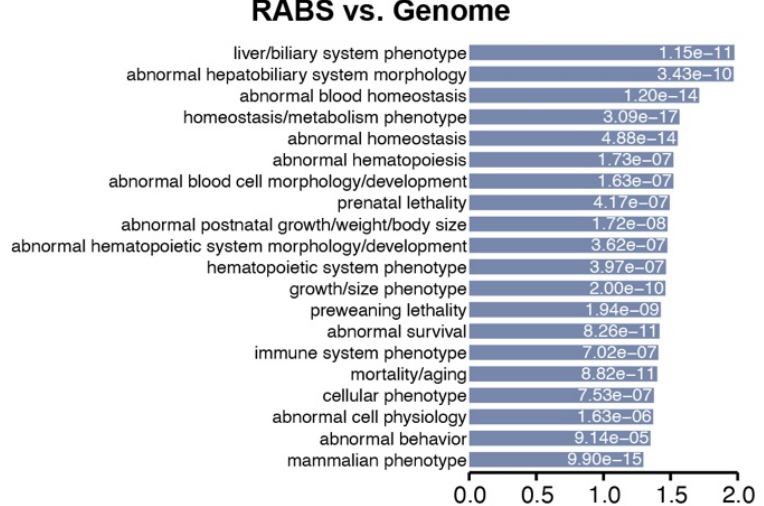

Hypergeometric Fold Enrichment
C

Non-RABS E-Box vs. OC

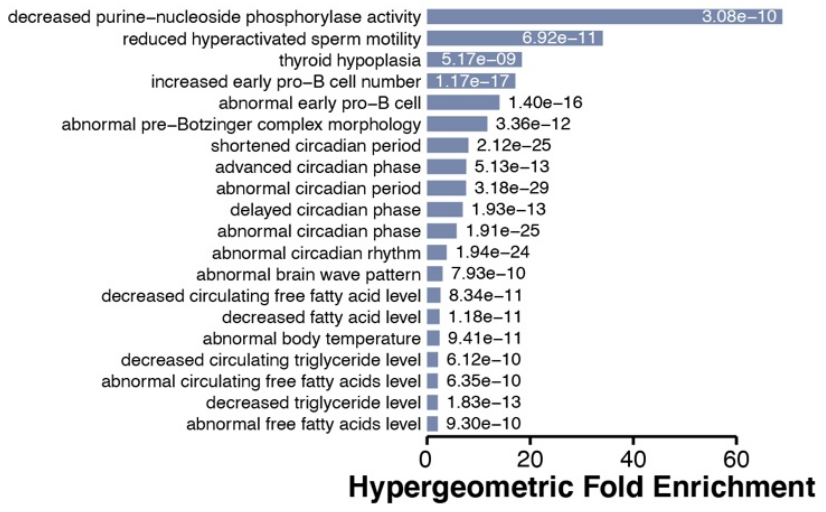

D

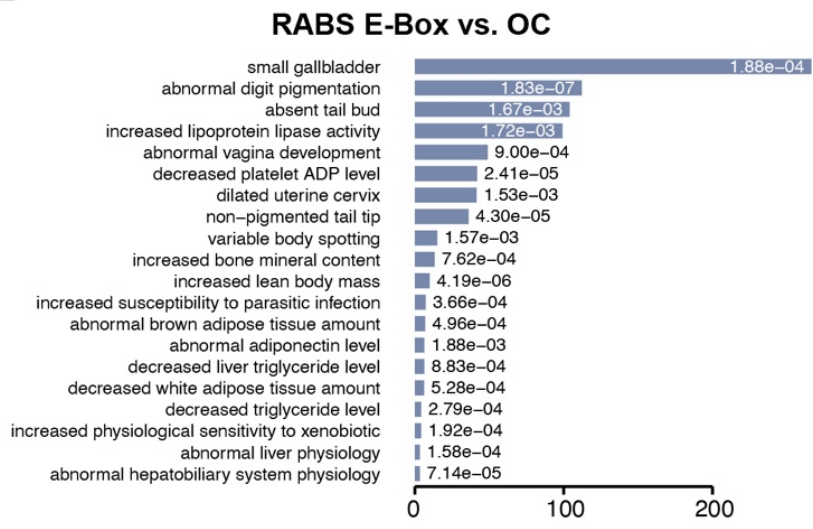

Hypergeometric Fold Enrichment

Figure S4: RABS-proximal genes are enriched for more lineage-specific phenotypic

1171 associations than Non-RABS-proximal genes.

1172 (A)Phenotypic association enrichment of Non-RABS E-Box motifs using the entire mouse

1173 genome as background.

1174 (B) Phenotypic association enrichment of RABS E-Box motifs using the entire mouse

1175 genome as background.

1176 (C) Phenotypic association enrichment of Non-RABS E-Box motifs using open chromatin

1177 regions defined by DNase accessibility at any timepoint as background

1178 (D) Phenotypic association enrichment of RABS E-Box motifs using open chromatin regions

1179 defined by DNase accessibility at any timepoint as background 
bioRxiv preprint doi: https://doi.org/10.1101/2020.11.09.375469; this version posted November 10,2020 . The copyright holder for this preprint (which was not certified by peer review) is the author/funder, who has granted bioRxiv a license to display the preprint in perpetuity. It is made available under aCC-BY 4.0 International license.

1180 Enrichments were calculated by the tool GREAT using the two nearest genes within $1 \mathrm{Mb}$ of

1181 each E-Box motif. FDR corrected hypergeometric q-values are either overlaid or adjacent to each

1182 bar.

1183 


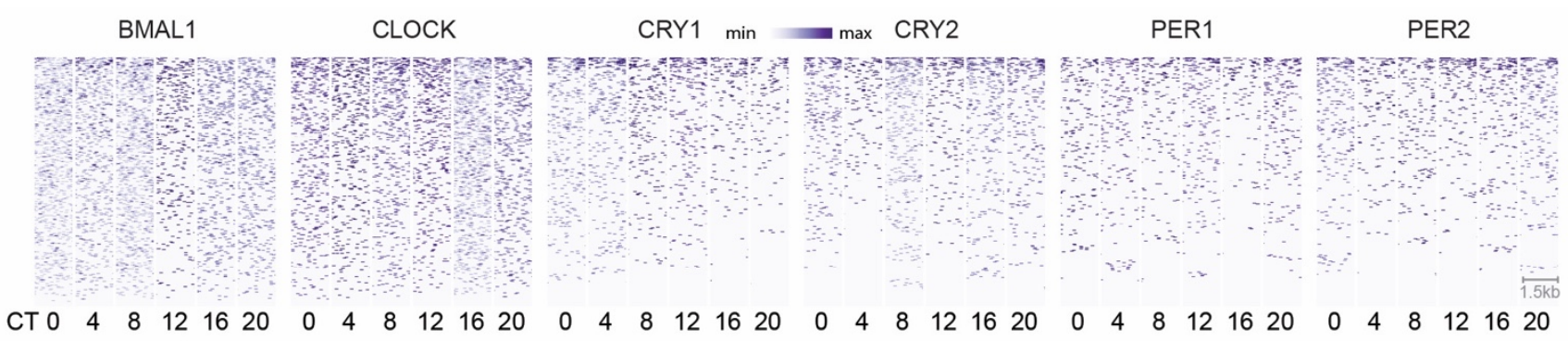

1185 Figure S5: RSINE1 elements not in open chromatin do not display oscillatory CR ChIP-seq

1186 signal.

1187 ChIP-seq signal for each CR across circadian time (CT) at a randomly selected set of 328

1188 RSINE1 elements which are not bound by CRs and do not reside in open chromatin regions as

1189 defined by DNase-seq. Heatmaps are centered at the middle of each element and extend $750 \mathrm{bp}$

1190 in each direction, and are sorted by mean signal intensity across all rows for a given CR.

1191 Color scaling is relative to min-max signal within each block of heatmaps. 

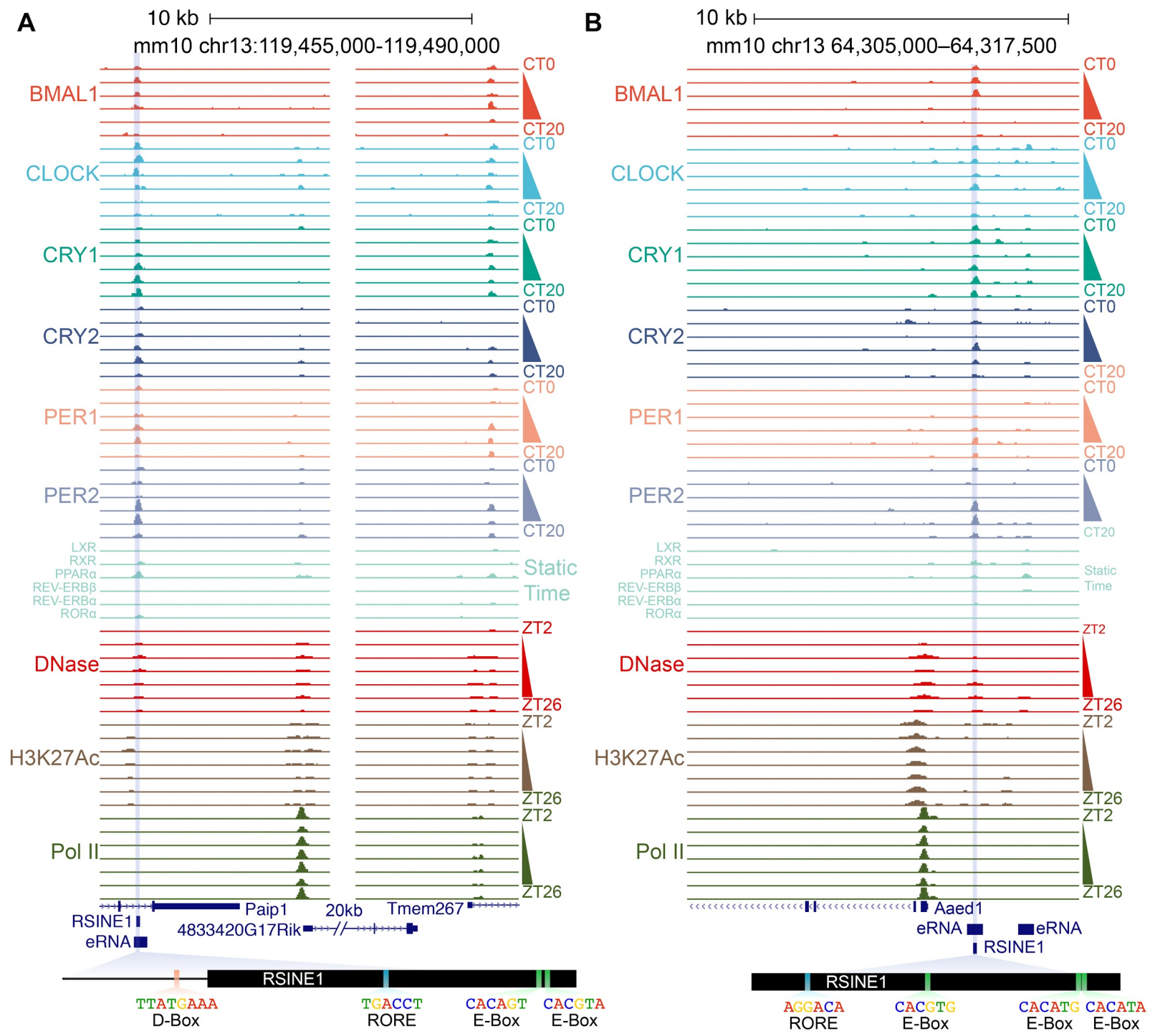

1193 Figure S6: UCSC genome browser screenshots of the two RSINE1s selected for luciferase

1194 reporter assays.

$1195 \quad$ (A)Browser shot of the Tmem267 locus.

1196 (B) Browser shot of the Aaed1 locus.

1197 In each panel, coordinates of oscillating eRNAs are shown alongside the location of an

1198 overlapping CR bound RSINE1. The location of the RSINE1 cloned into the luciferase reporter

1199 in Fig 7 is highlighted, and the motif content of that particular element is shown. The Tmem 267 
bioRxiv preprint doi: https://doi.org/10.1101/2020.11.09.375469; this version posted November 10,2020 . The copyright holder for this preprint (which was not certified by peer review) is the author/funder, who has granted bioRxiv a license to display the preprint in perpetuity. It is made available under aCC-BY 4.0 International license.

1200 RSINE1 shows a small upstream region where a D-Box motif was found adjacent to the insertion

1201 site.

1202 